\title{
Truthful Mechanisms with Implicit Payment Computation
}

MOSHE BABAIOFF, Microsoft Research, Herzeliya, Israel.

ROBERT D. KLEINBERG, Computer Science Department, Cornell University, Ithaca, NY, USA. ALEKSANDRS SLIVKINS, Microsoft Research, New York, NY, USA.

First version: April 2010

This version: November 2015

It is widely believed that computing payments needed to induce truthful bidding is somehow harder than simply computing the allocation. We show that the opposite is true: creating a randomized truthful mechanism is essentially as easy as a single call to a monotone allocation rule. Our main result is a general procedure to take a monotone allocation rule for a single-parameter domain and transform it (via a black-box reduction) into a randomized mechanism that is truthful in expectation and individually rational for every realization. The mechanism implements the same outcome as the original allocation rule with probability arbitrarily close to 1 , and requires evaluating that allocation rule only once. We also provide an extension of this result to multi-parameter domains and cycle-monotone allocation rules, under mild star-convexity and non-negativity hypotheses on the type space and allocation rule, respectively.

Because our reduction is simple, versatile, and general, it has many applications to mechanism design problems in which re-evaluating the allocation rule is either burdensome or informationally impossible. Applying our result to the multi-armed bandit problem, we obtain truthful randomized mechanisms whose regret matches the information-theoretic lower bound up to logarithmic factors, even though prior work showed this is impossible for truthful deterministic mechanisms. We also present applications to offline mechanism design, showing that randomization can circumvent a communication complexity lower bound for deterministic payments computation, and that it can also be used to create truthful shortest path auctions that approximate the welfare of the VCG allocation arbitrarily well, while having the same running time complexity as Dijkstra's algorithm.

Categories and Subject Descriptors: J.4 [Social and Behavioral Sciences]: Economics; K.4.4 [Computers and Society]: Electronic Commerce; F.2.2 [Analysis of Algorithms and Problem Complexity]: Nonnumerical Algorithms and Problems

General Terms: theory, algorithms, economics

Additional Key Words and Phrases: algorithmic mechanism design, single-parameter mechanisms, multiarmed bandits, regret, multi-parameter mechanisms

This is a merged and revised version of the conference papers [Babaioff et al.2010, 2013] that have appeared in the ACM Conf. on Electronic Commerce (ACM EC) in 2010 and 2013, respectively. This paper contains all results from [Babaioff et al. 2010] and the main result from [Babaioff et al. 2013] (in Section[8]. This version is updated to reflect the current status of the follow-up work and open questions.

Parts of this research have been done while R. Kleinberg was a Consulting Researcher at Microsoft Research Silicon Valley. He was also supported by NSF Awards CCF-0643934 and AF-0910940, an Alfred P. Sloan Foundation Fellowship, and a Microsoft Research New Faculty Fellowship. 


\section{INTRODUCTION}

Algorithmic Mechanism Design studies the problem of implementing the designer's goal under computational constraints. Multiple hurdles stand in the way for such implementation. Computing the desired outcome might be hard (as in the case of combinatorial auctions) or truthful payments implementing the goal might not exist (as when exactly minimizing the make-span in machine scheduling [Archer and Tardos 2001]). Even when payments that will generate the right incentives do exist, finding such payments might be computationally costly or impossible due to online constraints.

It is widely believed that computing payments needed to induce truthful bidding is somehow harder than simply computing the allocation. For example, the formula for payments in a VCG mechanism involves recomputing the allocation with one agent removed in order to determine that agent's payment; this seemingly increases the required amount of computation by a factor of $n+1$, where $n$ is the number of agents. Likewise, for truthful single-parameter mechanisms the formula for payments of a given agent includes integrating the allocation rule over this agent's bid [Mverson 1981; Archer and Tardos 2001]. In some contexts with incomplete observable information, such as online pay-per-click auctions, computing these "counterfactual allocations" may actually be information-theoretically impossible. This calls into question the mechanism designer's ability to compute payments that make an allocation rule truthful, even when such payment functions are known to exist. Rigorous lower bounds based on these observations have been established for the communication complexity [Babaioff et al. 2013] and regret [Babaioff et al. 2014; Devanur and Kakade 2009] of truthful deterministic mechanisms.

In contrast to these negative results, we show that the opposite is true for randomized single-parameter mechanisms that are truthful-in-expectation: computing the allocation and payments is essentially as easy as a single call to the allocation rule. This allows for positive results that circumvent the lower bounds for deterministic mechanisms cited earlier.

\subsection{Single-parameter mechanisms}

We consider an arbitrary single-parameter domain. The paradigmatic example is an auction that allocates items between agents whose utility is linear in the number of items they receive. The private information of each agent is expressed by a single parameter: her value per item 1 Each agent submits a bid, then the mechanism performs the allocation and charges payments. A mechanism is called "truthful" if each agent maximizes her utility by submitting her true value per item. The allocation rule in a truthful mechanism is called "truthfully implementable". It is known that an allocation rule is truthfully implementable if and only if it is "monotone": increasing one agent's bid while keeping all other bids the same does not decrease this agent's allocation [Mverson 1981; Archer and Tardos 2001]. A similar property holds for randomized mechanisms and truthfulness-in-expectation.

Our contributions. Our main result is a general procedure to take any monotone-inexpectation allocation rule $\mathcal{A}$ and transform it into a randomized mechanism that is truthful-in-expectation, implements the same outcome as $\mathcal{A}$ with probability arbitrarily close to 1 , and requires evaluating that allocation rule only once. (We refer to this

\footnotetext{
${ }^{1}$ In a general single-parameter domain, the allocation rule selects an outcome from some arbitrary collection of feasible outcomes. Each agent has her own type of "good", and for each agent there is an arbitrary, publicly known mapping from feasible outcomes to a real-valued amount of the corresponding good. The agent's utility is linear in this amount; the value per unit amount of good is her private information.
} 
procedure as the generic transformation.) The allocation rule $\mathcal{A}$ is accessed only as a function call, so our result applies even if $\mathcal{A}$ is an online algorithm. Moreover, for each realization of randomness an agent never loses by participating in the mechanism and bidding truthfully; thus the agents are protected from undesirable random deviations.

We make a distinction between randomness in the mechanism and randomness in "nature": the environment that the mechanism interacts with. Randomness in nature is subject to modeling assumptions and hence is less "reliable"; moreover, agents' beliefs about nature may be different from the mechanism's. On the other hand, randomness in the mechanism is fully controlled by the mechanism. Therefore it is desirable to design mechanisms that are truthful in a stronger sense: in expectation over the mechanism's random seed, for every realization of randomness in nature; we will call such mechanisms ex-post truthful. It is easy to see from [Myerson 1981; Archer and Tardos 2001] that in any ex-post truthful mechanism the allocation rule must satisfy ex-post monotonicity (which is defined similarly to ex-post truthfulness). In the generic transformation described above, if the original allocation rule $\mathcal{A}$ is ex-post monotone then the resulting randomized mechanism is ex-post truthful.

Similarly, our result extends to Bayesian incentive-compatibility: if $\mathcal{A}$ is monotone in expectation with respect to a Bayesian prior over other agents' bids, then the mechanism is truthful in expectation over this prior.

Our generic transformation is particularly useful for mechanism design problems in which re-evaluating the allocation rule is either burdensome or informationtheoretically impossible.

\subsection{Bandit mechanisms}

A leading problem for which only a single call to the allocation rule can be evaluated is the multi-armed bandit (MAB) mechanism design problem [Babaioff et al. 2014; Devanur and Kakade 2009]. In this problem information about the state of the world is dynamically revealed during the allocation; the particular information that is revealed depends on the prior choices of the allocation, and in turn may impact the future choices. Simulating the allocation rule on different inputs may therefore require information that was not observed on the actual run. This "informational obstacle" (insufficient observable information) is a crucial obstacle for deterministic ex-post truthful MAB mechanisms; it is used in [Babaioff et al. 2014] to derive that the appropriate payments cannot be computed unless the allocation rule is very "naïve" (and therefore suboptimal).

To put more context, MAB mechanisms are motivated by online pay-per-click ad auctions, and were suggested in [Babaioff et al. 2014; Devanur and Kakade 2009] as a simple model which combines strategic bidding by agents and online learning by the mechanism. Each agent has a single ad that she wants to display to users, and derives utility only if her ad is clicked. The value per click is her private information. The allocation rule proceeds in rounds: in each round the mechanism allocates one ad to be shown to a user and observes whether this ad was clicked. The click probabilities (also known as "click-through rates", or CTRs) are unknown to the mechanism, and need to be estimated during the run of the allocation rule. All bids are submitted before the allocation starts, and all payments are assigned after it ends.

MAB mechanisms are related to MAB algorithms: the allocation rule is essentially an MAB algorithm whose "rewards" are clicks weighted by the corresponding bids. Moreover, welfare of an MAB mechanism is precisely the same as the total reward of its allocation rule 2 Therefore one could directly compare the performance of truthful

${ }^{2}$ This is because payments cancel out: the total amount paid by the agents is equal to the total amount received by the mechanism. 
MAB mechanisms with that of MAB algorithms; both can be quantified using regret: the loss in welfare compared to the benchmark which always picks the best ad.

Following [Babaioff et al.|2014; Devanur and Kakade|2009], we focus on the stochastic version of the problem, i.e. we assume that the CTRs do not change over time. Then the "randomness in nature" corresponds to the random clicks, and ex-post truthfulness means truthfulness for every realization of the clicks (but in expectation over the randomness in the mechanism). Note that ex-post truthfulness is a very strong property which holds even if the clicks are chosen by an oblivious adversary. As discussed in [Babaioff et al. 2014; Devanur and Kakade 2009], this property is highly desirable, compared to the weaker notion of "truthfulness in expectation over clicks", even if the corresponding mechanism has regret guarantees that only apply to the stochastic setting.

Our contributions. Applying our generic transformation to the MAB problem we derive that the problem of designing truthful MAB mechanisms reduces to the problem of designing monotone MAB allocation rules. Such a problem has not been previously studied in the rich literature on MAB.

Our main result in this direction is a randomized MAB mechanism that is ex-post truthful and has regret $O\left(T^{1 / 2}\right)$ for the stochastic version. This upper bound on regret matches the information-theoretic lower bound for algorithms in the same setting (i.e., the lower bound holds even in the absence of incentive constraints). This stands in contrast to the lower bound of [Babaioff et al. 2014], where it was shown that deterministic ex-post truthful MAB mechanisms must suffer a larger regret of $\Omega\left(T^{2 / 3}\right)$.

On a technical level, we design a new MAB allocation rule that is ex-post monotone and has regret $O\left(T^{1 / 2}\right)$ for the stochastic setting. (We use it to obtain a randomized ex-post truthful MAB mechanism with the same regret.) Moreover, we show that UCB1 [Auer et al. 2002a] (and a number of similar MAB algorithms) give rise to MAB allocations that are monotone in expectation over clicks, and therefore can be transformed to randomized $\mathrm{MAB}$ mechanisms that are truthful in the same sense and have optimal regret.

The new ex-post monotone MAB allocation rule is deterministic, which rigorously confirms the intuition from [Babaioff et al. 2014; Devanur and Kakade 2009] that the impossibility results for deterministic MAB mechanisms are caused by the "informational obstacle" (insufficient observable information about clicks) rather than ex-post monotonicity.

\subsection{Other contributions}

Power of randomization. As a by-product of our analysis of MAB mechanisms, we obtain an unconditional separation between the power of randomized vs. deterministic ex-post truthful mechanisms for welfare maximization, in the online setting. (The separation result is unconditional in the sense that it considers exactly the same setting for both classes of mechanisms.) This complements the result of Dobzinski and Dughmi [2009], which gives a separation between these two classes of mechanisms in the offline setting, under a polynomial communication complexity constraint. It is worth noting that the separation in Dobzinski and Dughmi [2009] applies to a rather unnatural problem (two-player multi-unit auctions in which if at least one item is allocated, then all items are allocated and each player receives at least one item) whereas our separation result is for a natural problem: online pay-per-click ad auctions for a single slot, with unknown click-through rates.

For the objective of revenue maximization, separations between randomized and deterministic mechanisms have been known for much longer [Thanassoulis 2004; Manelli and Vincent 2006; Dobzinski et al. 2012; Briest et al. 2014] and are in some 
sense less surprising. Randomization allows the mechanism to access a larger set of possible allocations, i.e. the set of all probability distributions over pure allocations, and in some cases this leads to greater revenue, for example by permitting more finegrained price discrimination between agent types. This is not the case for the objective of maximizing welfare (because VCG mechanisms are deterministic and they maximize welfare pointwise while obeying incentive constraints). For welfare maximization, randomized mechanisms are sometimes more powerful than deterministic ones due to other reasons, such as computational power or informational limitations (as in the problem we study).

Offline mechanisms. Our main result also has implications for offline mechanism design. Nisan and Ronen, in their seminal paper [Nisan and Ronen 2001] which started the field of algorithmic mechanism design, cite the apparent $n$-fold computational overhead of computing VCG payments and pose the open question of whether payments can be computed faster than solving $n$ versions of the original problem, e.g. for VCG path auctions. Our result shows that the answer is affirmative, if one adopts the truthful-in-expectation solution concept and tolerates a mechanism that outputs an outcome whose welfare is a $(1+\epsilon)$-approximation to that of the VCG allocation, for arbitrarily small $\epsilon>0$. Babaioff et al. [2013] present a social choice function $f$ in an $n$-player single-parameter domain such that the deterministic communication complexity required for truthfully implementing $f$ exceeds that required for evaluating $f$ by a factor of $n$. Our result shows that no such lower bound holds when one considers randomized mechanisms, again allowing for a small amount of random error in the allocation.

Extension to multi-parameter mechanisms. We extend our generic transformation from single-parameter to multi-parameter mechanisms. It is known that a multiparameter allocation rule is truthfully implementable if and only if it satisfies a property called "cycle-monotonicity". (This is a rather strong property which specializes to monotonicity in the single-parameter case.) Similar to the single-parameter case, we present a general procedure to take any cycle-monotone allocation rule $\mathcal{A}$ and transform it into a randomized mechanism that is truthful-in-expectation, implements the same outcome as $\mathcal{A}$ with probability arbitrarily close to 1 , and requires evaluating that allocation rule only once. The technical contribution here is that we find a reduction from the multi-parameter setting to the single-parameter case.

While much more general that our single-parameter transformation, this result may be more difficult to apply. This is because cycle-monotonicity is known to be a very restrictive property. However, the follow-up work already provides two applications, see Section 2.1 for details.

\subsection{Map of the paper}

This paper makes four high-level contributions: the generic transformation for singleparameter mechanisms (Sections 4 and 5), the two applications to off-line mechanism design (Section 6), the results on MAB mechanisms (Section 7), and an extension to multi-parameter mechanisms (Section 8). Presenting these results requires a significant amount of preliminaries on mechanisms design (Section 3), multi-armed bandits (Section 7.1), and multi-parameter mechanism (Section 8.1). We conclude with open questions (Section 9).

A considerable amount of work followed up on the initial conference publication [Babaioff et al. 2010] of this paper. This work is discussed in Section 2.1. 


\section{RELATED WORK AND FOLLOW-UP WORK}

The characterization of truthful mechanisms for single-parameter domains, given by Myerson [1981] for single-item auctions and by Archer and Tardos [2001] for a more general class of single-parameter problems, states that a mechanism is truthful if and only if its allocation rule is monotone and its payment rule charges each agent its value for the realized outcome, minus a correction term expressed as an integral over all types lower than the agent's declared type. Exact computation of this correction term may be intractable, but Archer et al. [2004] developed a clever workaround: one can use random sampling to compute an unbiased estimator of the correction term, at the cost of evaluating the allocation rule once more. Thus, for $n$ agents, the allocation rule must be evaluated $n+1$ times: once to determine the actual allocation, and once more per agent to determine that agent's payment. Our generic transformation relies on a generalization of this random sampling technique, but we show how to avoid recomputing the allocation rule when determining each agent's payment, by coupling payment generation with the allocation itself.

The question of whether computing payments is computationally harder than computing the allocation was raised by Nisan and Ronen [2001] in the context of VCG path auctions. The most significant progress to date was the communication complexity lower bound of Babaioff et al. [2013] mentioned above.

Payment computation in online mechanism design is a central issue in the analysis of truthful MAB mechanisms in Babaioff et al. [2014] and Devanur and Kakade [2009]. The main result of [Babaioff et al. 2014] is a characterization of deterministic ex-post truthful mechanisms. It is more restrictive than the Myerson and ArcherTardos characterization. The reason is that computing an agent's payment requires knowing how many clicks she would have received if she had submitted a lower bid value, which may require the mechanism to hypothetically go back into the past and allocate impressions to a different agent for the purpose of seeing whether a user would have clicked on that agent's advertisement. Such counterfactual information is typically impossible to obtain in an online setting.

Babaioff et al. [2014] focus on welfare maximization. Using the above characterization, they prove that any deterministic ex-post truthful MAB mechanism must incur regret $\Omega\left(T^{2 / 3}\right)$, whereas MAB algorithms for the same setting can achieve regret $O\left(T^{1 / 2}\right)$. Devanur and Kakade [2009] consider revenue maximization, and derive a similar $\Omega\left(T^{2 / 3}\right)$ lower bound on loss of revenue compared to the VCG payments 3

Dynamic auctions |Athey and Segal 2013; Bergemann and Välimäki 2010; Bergemann and Said 2011] constitute another setting in which information is revealed "dynamically" (over time). However, while in MAB auctions all information from the agents (the bids) is submitted only once and then information is revealed to the mechanism by the environment over time, in dynamic auctions the agents continuously observe private "signals" from the environment and submit "actions" to the mechanism. Accordingly, providing the right incentives becomes much more challenging. On the other hand, existing work has focused on a fully Bayesian setting with known priors on the signals, whereas all of our results do not rely on priors.

Finally, several recent papers have explored the theme of reductions in algorithmic mechanism design. Unlike our work which requires mechanisms to be truthful for every realization of the agents' types, these papers focus on Bayesian settings and adopt Bayesian incentive-compatibility as their solution concept. A reduction converting any allocation rule into a Bayesian incentive-compatible mechanism with approx-

\footnotetext{
${ }^{3}$ For revenue-maximizing $\mathrm{MAB}$ mechanisms, there is no clear comparison with the performance of MAB algorithms.
} 
imately the same expected social welfare was developed in [Hartline and Lucier 2010; Bei and Huang 2011; Hartline et al. 2011]. Chawla et al. [2012] considered black-box reductions of mechanism design problems to algorithmic problems with the same objective, and demonstrated significant limitations of this approach. The breakthrough results of Cai et al. [2012; 2013a; 2013b] and Daskalakis and Weinberg [2014] circumvented these limitations by instead reducing to algorithmic problems with a modified objective. In particular, reductions from revenue-maximizing mechanisms to welfaremaximizing algorithms are presented in [Cai et al. 2012, 2013a], whereas Cai et al. [2013b] and Daskalakis and Weinberg [2014] present reductions for non-linear objective functions, such as makespan in scheduling:

\subsection{Follow-up work (subsequent to [Babaioff et al. 2010])}

Our generic transformation exhibits high variability in payments, and includes an explicit tradeoff between the variability in payments and the loss in performance. Formally, variability can be expressed as variance, maximal absolute value, or (for positive types) maximal rebate. Performance can be expressed as welfare or revenue. Wilkens and Sivan [2012] have proved this tradeoff to be optimal in a certain worstcase sense: our transformation achieves the optimal worst-case variance in payments for any given worst-case loss in performance, where the worst case is over all monotone allocation rules. Their result applies to any single-parameter domain and any of the above notions of variability and performance.

Our generic transformation is likely to be very useful in single-parameter settings which exhibit the "informational obstacle" (insufficient observable information) such as the one found for deterministic MAB mechanisms. The follow-up work describes three additional settings. First, Wilkens and Sivan [2012] observe that the same obstacle arises in offline pay-per-click ad auctions with multiple ad slots, where the CTRs have slot-specific multipliers. In conjunction with our generic transformation, an obvious welfare-maximizing allocation rule for that setting results in a truthfulin-expectation mechanism. Second, Shnayder et al. [2012] describe a packet scheduling problem in a network router, where the "informational obstacle" arises due to the potentially missing information about packet arrival times. (As they observe, this information may be missing not only because it is not observed by the router but also because the router simply does not have space to store it.) They design a monotone allocation rule for their setting, and use our generic transformation to convert it to a truthful-in-expectation mechanism. Third, Gatti et al. [2012] consider an extension of MAB mechanisms to multiple ad slots. While they provide truthful mechanisms based on the simple MAB mechanism from [Babaioff et al. 2014; Devanur and Kakade 2009], our generic transformation could give rise to more efficient truthful mechanisms.

Wilkens and Sivan [2012] obtain a similar "single-call reduction" (i.e., a reduction from allocation rules to truthful-in-expectation mechanisms which calls the allocation rule only once) for multi-parameter allocation rules that are maximal-indistributional-range (MIDR). MIDR allocation rules [Dobzinski and Dughmi 2009] pick a welfare-maximizing distribution over outcomes from some fixed collection of distributions; they are precisely the allocation rules for which VCG payments produce a truthful mechanism. This result is an independent work with respect to, and a special case of, the multi-parameter reduction in Section 8 .

The multi-parameter generic transformation in Section 8 has been used in two recent papers. First, Jain et al. [2011] used it to speed up the payment computation for a mechanism that allocates batch jobs in a cloud system. Second, Huang and Kannan

\footnotetext{
${ }^{4}$ All papers discussed in this paragraph, except [Hartline and Lucier 2010], have appeared after the conference publication of this paper [Babaioff et al. [2010].
} 
[2012] used it to compute payments for their privacy-preserving procurement auction for spanning trees, which is based on the well-known "exponential privacy mechanism" from prior work [McSherry and Talwar 2007].

Simplified payment computation. Our generic transformation is most useful if the allocation rule cannot be invoked more than once, as in "bandit mechanisms" or other examples provided in follow-up work. Segal [2010] has observed that any truthful single-parameter mechanism can be implemented in a much simpler way, as long as two calls to the allocation rule are allowed: one computes the allocation, and the other one generates random payments with the correct expectation. In the first call one uses the original bids. For the second call, one selects an agent uniformly at random, and uses the random sampling trick from Archer and Tardos [2001] described above to compute the payment for this agent, and then scales the payment appropriately 5

Also, a simpler generic transformation is possible if one settles for a weaker notion of Bayesian incentive-compatibility [Hartline 2012].

\section{PRELIMINARIES}

Single-parameter domains. We present the single parameter model for which we apply our procedure. The model is very similar to the model of Archer and Tardos [Archer and Tardos 2001], yet it is slightly more general. We state the model is terms of values and not costs and allow the values to be both positive and negative. We also allow randomization by nature. All these changes are minor and do not change the fundamental characterization, yet are helpful to later derive our results.

Let $n$ be the number of agents and let $N=[n]$ be the set of agents. Each agent $i \in N$ has some private type consisting of a single parameter $x_{i} \in \mathcal{T}_{i}$ that describes the agent, and is known only to $i$, everything else is public knowledge. We assume that the domain $\mathcal{T}_{i}$ is an open subset of $\mathbb{R}$ which is an interval with positive length (possibly starting from $-\infty$ or going up to $\infty$ ). Let $\mathcal{T}=\mathcal{T}_{1} \times \mathcal{T}_{2} \times \ldots \times \mathcal{T}_{n}$ denote the domain of types and let $t \in \mathcal{T}$ denote the vector of true types.

There is some set of outcomes $\mathcal{O}$. For single-parameter domains, agents evaluate outcomes in a particular way that we describe next. For each agent $i \in N$ there is a function $a_{i}: \mathcal{O} \rightarrow \mathbb{R}_{+}$specifying the allocation to agent $i$. The value of an outcome $o \in \mathcal{O}$ for an agent $i \in N$ with type $x_{i}$ is $x_{i} \cdot a_{i}(o)$. The utility that agent $i \in N$ derives from outcome $o \in \mathcal{O}$ when he is charged $p_{i}$ is quasi-linear: $u_{i}=x_{i} \cdot a_{i}(o)-p_{i}$.

For instance, consider the allocation of $k$ identical units of good to agents with additive valuations: agent $i$ has a value of $x_{i}$ per unit. An outcome $o$ specifies how many items each agent receives: $a_{i}(o)$ is the number of items $i$ receives. His valuation for that outcome is his value per-unit times the number of units he receives.

A (direct revelation) deterministic mechanism $\mathcal{M}$ consists of the pair $(\mathcal{A}, \mathcal{P})$, where $\mathcal{A}: \mathcal{T} \rightarrow \mathcal{O}$ is the allocation rule and $\mathcal{P}: \mathcal{T} \rightarrow \mathbb{R}^{n}$ is the payment rule, i.e. the vector of payment functions $\mathcal{P}_{i}: \mathcal{T} \rightarrow \mathbb{R}$ for each agent $i$. Each agent is required to report a type $b_{i} \in \mathcal{T}_{i}$ to the mechanism, and $b_{i}$ is called the bid of agent $i$. We denote the vector of bids by $b \in \mathcal{T}$. The mechanism picks an outcome $\mathcal{A}(b)$ and charges agent $i$ payment of $\mathcal{P}_{i}(b)$. The allocation for agent $i$ when the bids are $b$ is $\mathcal{A}_{i}(b)=a_{i}(\mathcal{A}(b))$ and he is charged $\mathcal{P}_{i}(b)$. Agent $i$ 's utility when the agents bid $b \in \mathcal{T}$ and his type is $x_{i} \in \mathcal{T}_{i}$ is

$$
u_{i}\left(x_{i}, b\right)=x_{i} \cdot \mathcal{A}_{i}(b)-\mathcal{P}_{i}(b)
$$

\footnotetext{
${ }^{5}$ However, more work is needed for domains with negative agents' types, such as VCG shortest path auctions (see Section 6 for more details). In particular, one needs to carefully define the random sampling of the bid for payment computation, using a version of the argument in Section 5.2 to bound the loss in welfare.
} 
We also consider randomized mechanisms, which are distributions over deterministic mechanisms. For a randomized allocation rule $\mathcal{A}_{i}(b)$ and $\mathcal{P}_{i}(b)$ will denote the expected allocation and payment charged from agent $i$, when the bids are $b$. The expectation is taken over the randomness of the mechanism. Sometimes it will be helpful to explicitly consider the deterministic allocation and payment that is generated for specific random seed. in this case we use $w$ to denote the random seed and use $\mathcal{A}_{i}(b ; w)$ and $\mathcal{P}_{i}(b ; w)$ to denote allocation and payment when the seed is $w$.

There may be some outside randomization that influences the outcome and is not controlled by the mechanism, e.g. randomness in the realization of clicks in sponsored search auction. We call this randomization by nature. With such randomization $\mathcal{A}_{i}(b)$ and $\mathcal{P}_{i}(b)$ also encapsulate expectations over nature's randomization. Finally, we use the notation $\mathcal{A}_{i}(b ; w, r)$ and $\mathcal{P}_{i}(b ; w, r)$ to denote the allocation and payment charged from agent $i$, when the bids are $b$, the mechanism random seed is $w$ and nature's random seed is $r$.

Allocation and Mechanism Properties. Let $b_{-i}$ denote the vector of bids of all agents but agent $i$. We can now write the vector of bids as $b=\left(b_{-i}, b_{i}\right)$. Similar notation will be used for other vectors.

We next list two central properties, truthfulness and individual rationality.

- Mechanism $\mathcal{M}$ is truthful if for every agent $i$ truthful bidding is a dominant strategy: for every agent $i$, bidding $x_{i}$ always maximizes her utility, regardless of what the other agents bid. Formally,

$$
x_{i} \cdot \mathcal{A}_{i}\left(b_{-i}, x_{i}\right)-\mathcal{P}_{i}\left(b_{-i}, x_{i}\right) \geq x_{i} \cdot \mathcal{A}_{i}(b)-\mathcal{P}_{i}(b)
$$

holds for every agent $i \in N$, type $x_{i} \in \mathcal{T}_{i}$, bids of others $b_{-i} \in \mathcal{T}_{-i}$ and bid $b_{i} \in \mathcal{T}_{i}$ of agent $i$.

- Mechanism $\mathcal{M}$ is individually rational (IR) if an agent never receives negative utility by participating in the mechanism and bidding truthfully. Formally,

$$
x_{i} \cdot \mathcal{A}_{i}\left(b_{-i}, x_{i}\right)-\mathcal{P}_{i}\left(b_{-i}, x_{i}\right) \geq 0
$$

holds for every agent $i \in N$, type $x_{i} \in \mathcal{T}_{i}$ and bids of others $b_{-i} \in \mathcal{T}_{-i}$.

It will be helpful to establish terminology for the case that the above hold not only in expectation but also for specific realizations. For example, we will say that a mechanism is universally truthful if Equation (2) holds not only in expectation over the mechanism's randomness, but rather for every realization of that randomness. In general, every property that we define is defined by some inequality, and if the inequality holds for every realization of the mechanism randomness we say that it holds universally, and if it holds for every realization of nature randomness we say that it holds ex-post. When we want to emphasize that the property holds only in expectation over the nature's randomness we say that it holds stochastically.

Note that in an individually rational mechanism an agent is ensured not to incur any loss in expectation. That is rather unsatisfying as for some realizations the agent might suffer a huge loss. It is more desirable to design mechanisms that are universally ex-post individually rational, that is a truthful agent should incur no loss for every bids of the others and every realization of the random events (not only in expectation).

If all types are positive, then in addition to individual rationality it is desirable that all agents are charged a non-negative amount; this is known as the no-positivetransfers property. Finally, the welfare of a truthful mechanism is defined to be the total utility $\sum_{i} x_{i} \cdot \mathcal{A}_{i}(t)$.

Characterization. The following characterization of truthful mechanisms, due to Archer and Tardos [Archer and Tardos 2001], is almost identical to the characteriza- 
tion presented by Myerson [Myerson 1981] for truthful mechanisms in the special case of single item auctions. The crucial property of an allocation that yields truthfulness is monotonicity, defined as follows:

Definition 3.1. Allocation rule $\mathcal{A}$ is monotone if for every agent $i \in N$, bids $b_{-i} \in \mathcal{T}_{-i}$ and two possible bids of $i, b_{i} \geq b_{i}^{-}$, we have $\mathcal{A}_{i}\left(b_{-i}, b_{i}\right) \geq \mathcal{A}_{i}\left(b_{-i}, b_{i}^{-}\right)$.

Recall that monotonicity of an allocation rule is also defined universally and/or ex-post.

We next present the characterization of truthful mechanisms. In the theorem statement, the expression $\mathcal{A}_{i}\left(b_{-i}, u\right)$ is interpreted to equal zero when $u \notin \mathcal{T}_{i}$.

THEOREM 3.2. [Myerson 1981; Archer and Tardos 2001] Consider an arbitrary single-parameter domain. An allocation rule $\mathcal{A}$ admits a payment rule $\mathcal{P}$ such that the mechanism $(\mathcal{A}, \mathcal{P})$ is truthful if and only if $\mathcal{A}$ is monotone and moreover for each agent $i$ and bid vector $b$ it holds that $\int_{-\infty}^{b_{i}} \mathcal{A}_{i}\left(b_{-i}, u\right) d u<\infty$. In this case the payment $\mathcal{P}_{i}(b)$ for each agent $i$ must satisfy

$$
\mathcal{P}_{i}(b)=\mathcal{P}_{i}^{0}\left(b_{-i}\right)+b_{i} \mathcal{A}_{i}\left(b_{-i}, b_{i}\right)-\int_{-\infty}^{b_{i}} \mathcal{A}_{i}\left(b_{-i}, u\right) d u
$$

where $\mathcal{P}_{i}^{0}\left(b_{-i}\right)$ does not depend on $b_{i}$.

A mechanism is called normalized if for each agent $i$ and every bid vector $b$, zero allocation implies a zero payment: $\mathcal{A}_{i}(b)=0 \Rightarrow \mathcal{P}_{i}(b)=0$.

COROLlary 3.3. The truthful mechanism in Theorem 3.2 is normalized if and only if $\mathcal{P}_{i}^{0}\left(b_{-i}\right) \equiv 0$, in which case the mechanism is also individually rational and for positive-only types $\left(\mathcal{T} \subset \mathbb{R}_{+}^{n}\right)$ it moreover satisfies the no-positive-transfers property.

Both Theorem 3.2 and Corollary 3.3 hold in the "ex-post" sense (resp., "universal" sense), if $\mathcal{A}_{i}, \mathcal{P}_{i}$ and $\mathcal{P}_{i}^{0}\left(b_{-i}\right)$ are interpreted to mean their respective values for a specific random seed of nature (resp., mechanism). In Corollary 3.3, the mechanism is normalized in the same sense as it is truthful.

\section{THE GENERIC TRANSFORMATION FOR SINGLE-PARAMETER DOMAINS}

This section presents a generic procedure which takes any monotone allocation rule for a single-parameter domain and creates a randomized truthful-in-expectation mechanism which attains the same outcome as the original allocation rule with high probability. The resulting mechanism uses the allocation rule as a "black box," calls it only once, and allocates according to the this call. Henceforth, we will refer to this procedure as the generic transformation.

Our main result - the existence of the generic transformation with the desired properties - can be stated informally as follows.

THEOREM 4.1 (INFORMAL). Consider an arbitrary single-parameter domain with $n$ agents. Let $\mathcal{A}$ be a monotone allocation rule for this domain. Then for each $\mu \in[0,1]$ there exists a truthful mechanism $\mathcal{M}=(\widetilde{\mathcal{A}}, \widetilde{\mathcal{P}})$ with the following properties:

- $\mathcal{M}$ executes a single call to $\mathcal{A}(\tilde{b})$ to compute the allocation, with a pre-processing step to compute the modified bid vector $\tilde{b}$, and a post-processing step to compute the payments. Both pre- and post-processing steps take $O(n)$ time and do not depend on $\mathcal{A}$.

- For any bid vector $b$ and any fixed random seed of nature allocations $\widetilde{\mathcal{A}}(b)$ and $\mathcal{A}(b)$ are identical with probability at least $1-n \mu$.

$-\mathcal{M}$ is universally ex-post individually rational. If all types are positive, then $\mathcal{M}$ is ex-post no-positive-transfers, and never pays any agent $i$ more than $b_{i} \cdot \mathcal{A}_{i}(x) \cdot\left(\frac{1}{\mu}-1\right)$. 
Presenting the formal version of this result (Theorem 4.5) requires defining the generic transformation. We begin with an informal description thereof. As evidenced by Equation (4), the payment for agent $i$ is a difference of two terms: the agent's reported utility (i.e., the product of her bid and her allocation), minus the integral of the allocation assigned to every smaller bid value. We charge the agent for her reported utility, and we give her a random rebate whose expectation equals the required integral. When integrating a function over a finite interval, an unbiased estimator of the integral can be obtained by sampling a uniformly random point of that interval and evaluating the function at the sampled point. This idea was applied, in the context of mechanism design, by Archer et al. [2004]. Below, we show how to generalize the transformation to allow for integrals over unbounded intervals, as required by Equation (4). Using this transformation it is easy to transform any monotone allocation rule into a randomized mechanism that is truthful in expectation and only evaluates the allocation rule $n+1$ times: once to determine the actual allocation, and once more per agent to obtain an unbiased estimate of that agent's payment.

Our main innovation is a transformation that uses the same random sampling trick, but only needs to evaluate the allocation rule once during the entire mechanism. (In other words, it does not require additional calls to the allocation rule to compute the payments.) Assume that a parameter $\mu \in(0,1)$ is given. For every player, with probability $1-\mu$, we leave their bid unchanged; with probability $\mu$, we sample a smaller bid value. The allocation rule is invoked on these bids. An agent is always charged her reported value of the outcome, but if her bid was replaced with a smaller bid value then we refund her an amount equal to an unbiased estimator of the integral in Equation (4), scaled by $1 / \mu$ to counterbalance the fact that the refund is only being applied with probability $\mu$. A naïve application of this plan suffers from the following defect: the random resampling of bids modifies the expected allocation vector, so we need to obtain an unbiased estimator of the integral of the modified allocation rule. However, if we change our sampling procedure to obtain such an estimate, then this modifies the allocation rule once again, so we will still be estimating the wrong integral! What we need is a "fixed point" of this process of redefining the sampling procedure. Below, we give a definition of self-resampling procedures that satisfy the requisite fixed point property, and we give two simple constructions of self-resampling procedures.

A self-resampling procedure transforms the bid $b_{i}$ of a given agent $i$ bid into two correlated random values $\left(x_{i}, y_{i}\right)$, where $x_{i}$ is the modified bid presented to the original allocation rule, and $y_{i}$ is used (together with the allocation itself) in computing the payment for this agent. More specifically, $y_{i}$ is needed to correctly normalize the unbiased estimator of the integral in Equation (4) for the modified allocation rule, according to Theorem 4.2 below. For agents with positive types we define a simpler self-resampling procedure for which the unbiased estimator does not depend on $y_{i}$, and therefore, strictly speaking, the procedure only needs to output $x_{i}$ (more details can be found in Section 4.5). However, we explicitly return the $y_{i}$ even for the positive types so as to be consistent with the general definitions and (perhaps more importantly) because we use it to define self-resampling procedures with general support (see Section 4.4).

Thus, the formal description of our generic transformation consists of three parts:

(1) a method for estimating integrals by evaluating the integrand at a randomly sampled point,

(2) the definition and construction of self-resampling procedures,

(3) the generic transformation that uses the previous two ingredients to convert any monotone allocation rule into a truthful-in-expectation randomized mechanism.

We now specify the details of each of these three parts. 


\subsection{Estimating integrals via random sampling}

Let $I$ be a nonempty open interval in $\mathbb{R}$ (possibly with infinite endpoints) and let $g$ be a function defined on $I$. Let us describe a procedure for estimating the integral $\int_{I} g(z) d z$ by evaluating $g$ at a single randomly sampled point of $I$. The procedure is well known; we describe it here for the purpose of giving a self-contained exposition of our algorithm.

THEOREM 4.2. Let $F: I \rightarrow[0,1]$ be any strictly increasing function that is differentiable and satisfies $\inf _{z \in I} F(z)=0$ and $\sup _{z \in I} F(z)=1$. If $Y$ is a random variable with cumulative distribution function $F$, then

$$
\int_{I} g(z) d z=\mathbb{E}\left[\frac{g(Y)}{F^{\prime}(Y)}\right] .
$$

PROOF. Since $\inf _{z \in I} F(z)=0$ and $\sup _{z \in I} F(z)=1$, it follows that the random variable $Y$ is supported on the entire interval $I$. Our assumption that $F$ is differentiable implies that $Y$ has a probability density function, namely $F^{\prime}(z)$. Thus, for any function $h$, the expectation of $h(Y)$ is given by $\int_{I} h(z) F^{\prime}(z) d z$. Applying this formula to the function $h(z)=g(z) / F^{\prime}(z)$ one obtains the theorem.

\subsection{Self-resampling procedures}

The basic ingredient of our generic transformation is a procedure for taking a bid $b_{i}$ and a random seed $w_{i}$, and producing two random numbers $x_{i}\left(b_{i} ; w_{i}\right), y_{i}\left(b_{i} ; w_{i}\right)$. The mechanism will use $\left\{x_{i}\left(b_{i} ; w_{i}\right)\right\}_{i \in N}$ for determining the allocation and additionally $y_{i}\left(b_{i} ; w_{i}\right)$ for determining the payment it charges agent $i$. To prove that the mechanism is truthful in expectation we will require the following properties 6

Definition 4.3. Let $I$ be a nonempty interval in $\mathbb{R}$. A self-resampling procedure with support $I$ and resampling probability $\mu \in(0,1)$ is a randomized algorithm with input $b_{i} \in I$, random seed $w_{i}$, and output $x_{i}\left(b_{i} ; w_{i}\right), y_{i}\left(b_{i} ; w_{i}\right) \in I$, that satisfies the following properties:

(1) For every fixed $w_{i}, x_{i}\left(b_{i} ; w_{i}\right)$ and $y_{i}\left(b_{i} ; w_{i}\right)$ are non-decreasing functions of $b_{i}$.

(2) With probability $1-\mu, x_{i}\left(b_{i} ; w_{i}\right)=y_{i}\left(b_{i} ; w_{i}\right)=b_{i}$. Otherwise $x_{i}\left(b_{i} ; w_{i}\right) \leq y_{i}\left(b_{i} ; w_{i}\right)<$ $b_{i}$.

(3) The conditional distribution of $x_{i}\left(b_{i} ; w_{i}\right)$, given that $y_{i}\left(b_{i} ; w_{i}\right)=b_{i}^{\prime}<b_{i}$, is the same as the unconditional distribution of $x_{i}\left(b_{i}^{\prime} ; w_{i}\right)$. In other words,

$$
\operatorname{Pr}\left[x_{i}\left(b_{i} ; w_{i}\right)<a_{i} \mid y_{i}\left(b_{i} ; w_{i}\right)=b_{i}^{\prime}\right]=\operatorname{Pr}\left[x\left(b_{i}^{\prime} ; w_{i}\right)<a_{i}\right], \quad \forall a_{i} \leq b_{i}^{\prime}<b_{i} .
$$

(4) Consider the two-variable function

$$
F\left(a_{i}, b_{i}\right)=\operatorname{Pr}\left[y_{i}\left(b_{i} ; w_{i}\right)<a_{i} \mid y_{i}\left(b_{i} ; w_{i}\right)<b_{i}\right],
$$

which we will call the distribution function of the self-resampling procedure. For each $b_{i}$, the function $F\left(\cdot, b_{i}\right)$ must be differentiable and strictly increasing on the interval $I \cap\left(-\infty, b_{i}\right)$.

As it happens, it is easier to construct self-resampling procedures with support $\mathbb{R}_{+}$, and one such construction that we call the canonical self-resampling procedure (Algorithm 1) forms the basis for our general construction. We defer the discussion of self-resampling procedures with general support until after we have described and analyzed the generic transformation.

\footnotetext{
${ }^{6}$ To keep the notation consistent, we state Definition 4.3 for a given agent $i$. Strictly speaking, the subscript $i$ is not necessary.
} 


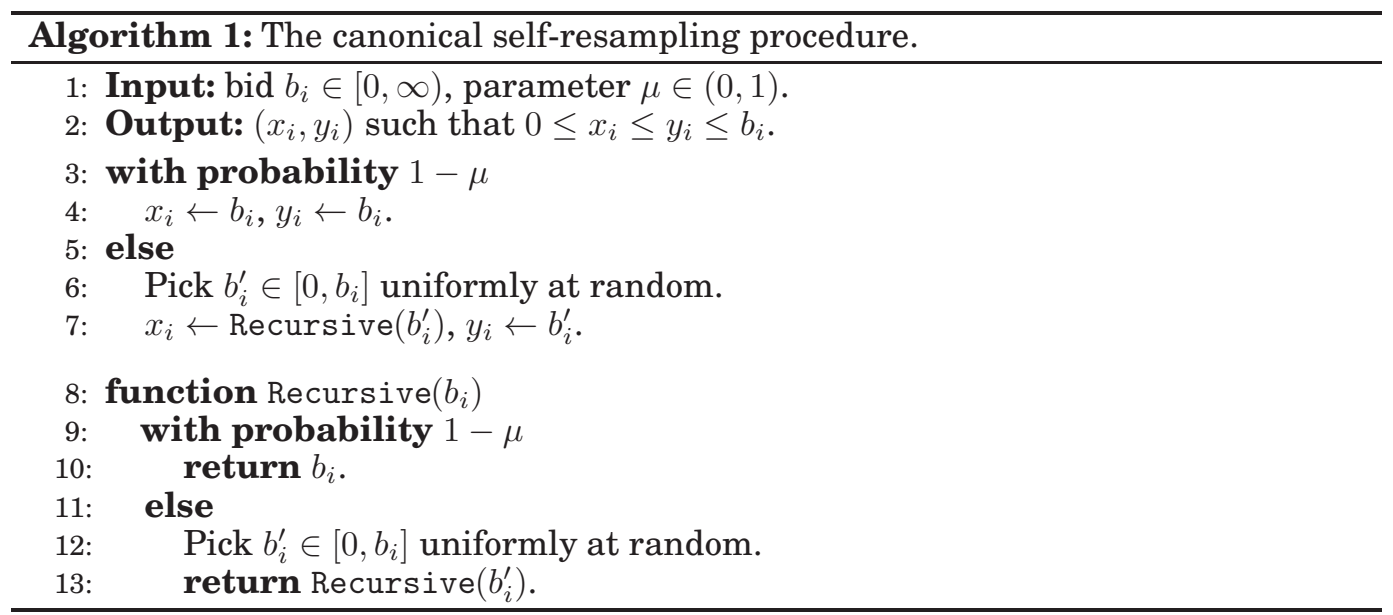

Proposition 4.4. Algorithm 1 is a self-resampling procedure with support $\mathbb{R}_{+}$ and resampling probability $\mu$. The distribution function for this procedure is $F\left(a_{i}, b_{i}\right)=$ $a_{i} / b_{i}$.

Proof. Properties 1 and 2 in Definition 4.3 are immediate from the description of the algorithm. The random seed $w_{i}$ for the algorithm can be defined as a countably infinite sequence of real numbers drawn independently and uniformly at random from $[0,1]$ interval. Then in order pick a random number in some range $[0, r]$, the algorithm takes the next number in this sequence and multiplies it by $r$.

Property 3 follows from the recursive nature of the sampling procedure: the event $y_{i}\left(b_{i} ; w_{i}\right)=b_{i}^{\prime}<b_{i}$ implies that the algorithm has followed the "else" branch on Line 5 , and has chosen $b_{i}^{\prime}$ in Line 6. Finally, the distribution function is $F\left(a_{i}, b_{i}\right)=a_{i} / b_{i}$ since conditional on the event $y_{i}\left(b_{i} ; w_{i}\right)<b_{i}$, the distribution of $y_{i}\left(b_{i} ; w_{i}\right)$ is uniform in the interval $\left[0, b_{i}\right]$. Property 4 follows trivially.

\subsection{The generic transformation}

Suppose we are given a monotone allocation rule $\mathcal{A}$ and for each agent $i \in N$ a self-resampling procedure that has resampling probability $\mu \in(0,1)$, support $\mathcal{T}_{i}$, and output values $f_{i}=\left(x_{i}, y_{i}\right)$. Let $F_{i}\left(a_{i}, b_{i}\right)$ denote the distribution function of the self-resampling procedure for agent $i$, and let $F_{i}^{\prime}\left(a_{i}, b_{i}\right)$ denote the partial derivative $\frac{\partial F_{i}\left(a_{i}, b_{i}\right)}{\partial a_{i}}$. Our generic transformation combines these ingredients into a randomized mechanism $\mathcal{M}=\operatorname{AllocToMech}(\mathcal{A}, \mu, \mathbf{f})$ that works as follows:

If $\mathcal{A}$ itself is randomized or if there is randomness arising from nature, then we allocate according to $\mathcal{A}(x ; w, r)$ and we assume that the algorithm's random seed $w$ and the nature's random seed $r$ are independent of the random seeds $w_{i}$ used in the resampling step.

We are now ready to present our main result:

THEOREM 4.5. Consider an arbitrary single-parameter domain. Let $\mathcal{A}$ be a monotone allocation rule. Suppose we are given an ensemble $\mathbf{f}$ of self-resampling procedures $f_{i}=\left(x_{i}, y_{i}\right)$ for each agent $i$, each with resampling probability $\mu \in(0,1)$. Then the mechanism $\mathcal{M}=(\widetilde{\mathcal{A}}, \widetilde{\mathcal{P}})=\operatorname{AllocToMech}(\mathcal{A}, \mu, \mathbf{f})$ has the following properties.

(a) $\mathcal{M}$ is truthful, universally ex-post individually rational, 
Mechanism 2: Generic transformation $\mathcal{M}=\operatorname{AllocToMech}(\mathcal{A}, \mu, \mathbf{f})$

1: Solicit bid vector $b \in \mathcal{T}$.

2: Execute each agent's self-resampling procedure using an independent random seed $w_{i}$, to obtain two vectors of modified bids

$$
\begin{aligned}
& x=\left(x_{1}\left(b_{1} ; w_{1}\right), \ldots, x_{n}\left(b_{n} ; w_{n}\right)\right), \\
& y=\left(y_{1}\left(b_{1} ; w_{1}\right), \ldots, y_{n}\left(b_{n} ; w_{n}\right)\right) .
\end{aligned}
$$

3: Allocate according to $\mathcal{A}(x)$.

4: Each agent $i$ is charged the amount $b_{i} \cdot \mathcal{A}_{i}(x)-R_{i}$, where $R_{i}$ is the rebate

$$
R_{i}= \begin{cases}\frac{1}{\mu} \cdot \frac{\mathcal{A}_{i}(x)}{F_{i}^{\prime}\left(y_{i}, b_{i}\right)} & \text { if } y_{i}<b_{i} \\ 0 & \text { otherwise }\end{cases}
$$

(b) For $n$ agents and any bid vector $b$ (and any fixed random seed of nature) allocations $\widetilde{\mathcal{A}}(b)$ and $\mathcal{A}(b)$ are identical with probability at least $1-n \mu$.

(c) If $\mathcal{T}=\mathbb{R}_{+}^{n}$ (all types are positive), and each $f_{i}$ is the canonical self-resampling procedure, then mechanism $\mathcal{M}$ is ex-post no-positive-transfers, and never pays any agent $i$ more than $b_{i} \cdot \mathcal{A}_{i}(x) \cdot\left(\frac{1}{\mu}-1\right)$.

Several remarks are in order.

- The mechanism never explicitly computes the payment for each agent $i$ (Equation (4)) but rather implicitly creates the correct expected payments through its randomization of the bids.

- The mechanism only invokes the original allocation rule $\mathcal{A}$ once. This property is very useful when it is impossible to invoke the allocation rule more than once, e.g. for multi-armed bandit allocations.

- The mechanism $\mathcal{M}$ is randomized even if $\mathcal{A}$ is deterministic. It is truthful in expectation over the randomness used by the self-resampling procedures.

- If $\mathcal{A}$ is ex-post monotone, then $\mathcal{M}$ will be ex-post truthful. To see this, fix nature's random seed $r$ and apply Theorem 4.5 to the allocation rule $\mathcal{A}_{r}$ induced by this $r$.

- If agents' types are positive then by part (b), the welfare of $\mathcal{M}$ is at least $1-n \mu$ times that of $\mathcal{A}$. Further results on bounding the welfare loss are presented in Section 5 .

- By definition of the payment rule, the mechanism is universally ex-post normalized. We will not explicitly mention this property in the subsequent applications.

Parameter $\mu$ controls the trade-off between the loss in welfare and the variance in payments, as quantified by the rebate size $R_{i}$. If $\mu$ is very small and the mechanism issues rebate(s), then its revenue may be very low and possibly negative. However, this risk may be mitigated if the auction maker runs many independent auctions, as may be the case in practice. Further, the follow-up paper [Wilkens and Sivan 2012] proves that our welfare vs. variance trade-off is optimal.

Proof of Theorem 4.5. We start with some notation. $\widetilde{\mathcal{A}}_{i}\left(b_{-i}, b_{i} ; q\right)$ denotes the allocation for agent $i$ given the bid vector $b=\left(b_{-i}, b_{i}\right)$ and the combined random seed $q=\left(w_{1}, \ldots, w_{n}, w, r\right)$. When we write $\widetilde{\mathcal{A}}_{i}\left(b_{-i}, u\right)$ without indicating the dependence on the $q$, we are referring to the unconditional expectation of $\widetilde{\mathcal{A}}_{i}\left(b_{-i}, u ; q\right)$ over $q$. 
To prove that $\mathcal{M}$ is truthful, we need to prove two things: that the randomized allocation rule $\widetilde{\mathcal{A}}$ is monotone, and that the expected payment rule $\widetilde{\mathcal{P}}$ satisfies

$$
\widetilde{\mathcal{P}}_{i}(b)=b_{i} \widetilde{\mathcal{A}}_{i}\left(b_{-i}, b_{i}\right)-\int_{-\infty}^{b_{i}} \widetilde{\mathcal{A}}_{i}\left(b_{-i}, u\right) d u .
$$

The monotonicity of randomized allocation rule $\widetilde{\mathcal{A}}$ follows from the monotonicity of $\mathcal{A}$ and the monotonicity property 1 in the definition of a self-resampling procedure. To prove that $\widetilde{\mathcal{P}}_{i}$ satisfies Equation (6), we begin by recalling that the payment charged to player $i$ is $b_{i} \mathcal{A}_{i}(x)-R_{i}$, where the rebate $R_{i}$ is defined by Equation (5). The expectation of $b_{i} \mathcal{A}_{i}(x)$ is simply $b_{i} \widetilde{\mathcal{A}}_{i}\left(b_{-i}, b_{i}\right)$, so to conclude the proof of truthfulness we must show that

$$
\mathbb{E}\left[R_{i}\right]=\int_{-\infty}^{b_{i}} \widetilde{\mathcal{A}}_{i}\left(b_{-i}, u\right) d u .
$$

Our proof of Equation (7) begins by observing that the conditional distribution of $x_{i}$, given that $y_{i}=u<b_{i}$, is the same as the unconditional distribution of $x_{i}\left(u ; w_{i}\right)$, by Property 3 of a self-resampling procedure. Combining this with the fact that the random seed $w_{i}$ is independent of $\left\{w_{j}: j \neq i\right\}$, we find that the conditional distribution of the tuple $x=\left(x_{-i}, x_{i}\right)$, given that $y_{i}=u$, is the same as the unconditional distribution of the vector $\hat{x}$ of modified bids that $\mathcal{M}$ would input into the allocation rule $\mathcal{A}$ if the bid vector were $\left(b_{-i}, u\right)$ instead of $\left(b_{-i}, b_{i}\right)$. Taking expectations, this implies that for all $u<b_{i}$, we have $\mathbb{E}\left[\mathcal{A}_{i}(x) \mid y_{i}=u\right]=\mathbb{E}\left[\mathcal{A}_{i}(\hat{x})\right]=\widetilde{\mathcal{A}}\left(b_{-i}, u\right)$.

Now apply Theorem 4.2 with the function $g(u)=\widetilde{\mathcal{A}}_{i}\left(b_{-i}, u\right)$. Recalling that $F_{i}\left(\cdot, b_{i}\right)$ is the cumulative distribution function of $y_{i}$ given that $y_{i}<b_{i}$, we apply the theorem to obtain

$$
\begin{aligned}
\int_{-\infty}^{b_{i}} \widetilde{\mathcal{A}}_{i}\left(b_{-i}, u\right) d u & =\mathbb{E}\left[\frac{\widetilde{\mathcal{A}}_{i}\left(b_{-i}, y_{i}\right)}{F_{i}^{\prime}\left(y_{i}, b_{i}\right)} \mid y_{i}<b_{i}\right]=\mathbb{E}\left[\frac{\mathcal{A}_{i}(x)}{F_{i}^{\prime}\left(y_{i}, b_{i}\right)} \mid y_{i}<b_{i}\right] \\
& =\mu \cdot \mathbb{E}\left[R_{i} \mid y_{i}<b_{i}\right],
\end{aligned}
$$

where the second equation follows from the equation derived at the end of the preceding paragraph, averaging over all $u<b_{i}$. Observing that $R_{i}=0$ unless $y_{i}<b_{i}$, an event that has probability $\mu$, we see that $\mathbb{E}\left[R_{i}\right]=\mu \cdot \mathbb{E}\left[R_{i} \mid y_{i}<b_{i}\right]$. Combined with Equation (8), this establishes Equation (7) and completes the proof that $\mathcal{M}$ is truthful.

Mechanism $\mathcal{M}$ is universally ex-post individually rational because agent $i$ is never charged an amount greater than $b_{i} \widetilde{\mathcal{A}}_{i}(b ; q)$. Part (b) follows from the union bound: the probability that $x_{i}=b_{i}$ for all $i$ is at least $1-n \mu$. For part (c), note that by Proposition 4.4, the canonical self-resampling procedure has distribution function $F\left(a_{i}, b_{i}\right)=a_{i} / b_{i}$, hence $F_{i}^{\prime}\left(y_{i}, b_{i}\right)=1 / b_{i}$, for all $i, y_{i}, b_{i}$. The rebate $R_{i}$ is equal either to 0 or to $\frac{1}{\mu} \cdot \frac{A_{i}(x)}{F_{i}^{\prime}\left(y_{i}, b_{i}\right)}=b_{i} \cdot A_{i}(x) \cdot \frac{1}{\mu}$. We also charge $b_{i} \cdot A_{i}(x)$ to agent $i$. The claimed upper bound on the amount paid to agent $i$ follows by combining these two terms.

\subsection{Self-resampling procedures with general support}

To construct a self-resampling procedure with support in an arbitrary interval $I$, we can use the following technique. Suppose $h:(0,1] \times I \rightarrow I$ is a two-variable function such that the partial derivatives $\partial h\left(z_{i}, b_{i}\right) / \partial z_{i}$ and $\partial h\left(z_{i}, b_{i}\right) / \partial b_{i}$ are well-defined and strictly positive at every point $\left(z_{i}, b_{i}\right) \in(0,1] \times I$. Suppose furthermore that $h\left(1, b_{i}\right)=b_{i}$ and $\inf _{z_{i} \in(0,1]}\left\{h\left(z_{i}, b_{i}\right)\right\}=\inf (I)$ for all $b_{i} \in I$. Then we define the $h$-canonical selfresampling procedure $\left(x_{i}^{h}, y_{i}^{h}\right)$ with support $I$, by specifying that

$$
\left\{\begin{array}{l}
x_{i}^{h}\left(b_{i} ; w_{i}\right)=h\left(x_{i}\left(1 ; w_{i}\right), b_{i}\right) \\
y_{i}^{h}\left(b_{i} ; w_{i}\right)=h\left(y_{i}\left(1 ; w_{i}\right), b_{i}\right),
\end{array}\right.
$$


where $\left(x_{i}, y_{i}\right)$ is the canonical self-resampling procedure as defined in Algorithm 1

Proposition 4.6. $\left(x_{i}^{h}, y_{i}^{h}\right)$ as defined in Equation (9) is a self-resampling procedure with support I and resampling probability $\mu$. The distribution function for $\left(x_{i}^{h}, y_{i}^{h}\right)$ is the unique two-variable function $F\left(a_{i}, b_{i}\right)$ such that

$$
h\left(F\left(a_{i}, b_{i}\right), b_{i}\right)=a_{i} \text { for all } a_{i}, b_{i} \in I, a_{i}<b_{i} .
$$

Proof. Property 1 in Definition 4.3 holds because of the monotonicity of $h$, Property 2 holds because $h\left(1, b_{i}\right)=b_{i}$ for all $b_{i}$, and Property 3 holds because the function $h$ is deterministic and monotone.

Let $F_{h}\left(a_{i}, b_{i}\right)$ and $F_{0}\left(a_{i}, b_{i}\right)$ be the distribution functions for the $h$-canonical and canonical self-resampling procedures, respectively. Recall that $F_{0}\left(a_{i}, b_{i}\right)=a_{i} / b_{i}$ by Proposition 4.4. Note that $F\left(a_{i}, b_{i}\right)$ in Equation (10) is unique (and hence well-defined) by the strict monotonicity of $h$.

The claim that $F_{h}\left(a_{i}, b_{i}\right)=F\left(a_{i}, b_{i}\right)$ easily follows from in Equation (9). By definition of $h$ we have

$$
h\left(y_{i}\left(1, w_{i}\right), b_{i}\right)<b_{i} \Longleftrightarrow y_{i}\left(1, w_{i}\right)<1 .
$$

Therefore, letting $y_{i}=y_{i}\left(1, w_{i}\right)$ we have

$$
\begin{aligned}
F_{h}\left(a_{i}, b_{i}\right) & \triangleq \operatorname{Pr}\left[h\left(y_{i}, b_{i}\right)<a_{i} \mid h\left(y_{i}, b_{i}\right)<b_{i}\right] \\
& =\operatorname{Pr}\left[y_{i}<F\left(a_{i}, b_{i}\right) \mid y_{i}<1\right] \\
& =F_{0}\left(F\left(a_{i}, b_{i}\right), 1\right) \\
& =F\left(a_{i}, b_{i}\right) .
\end{aligned}
$$

Our assumption that $h$ is differentiable and strictly increasing in its first argument now implies that the same property holds for $F$, which verifies Property 4 .

\subsection{A simplified generic transformation for positive types}

We focus on the important special case of positive types, and present Mechanism 3 a simplified version of the generic transformation (Mechanism 2), for this case.

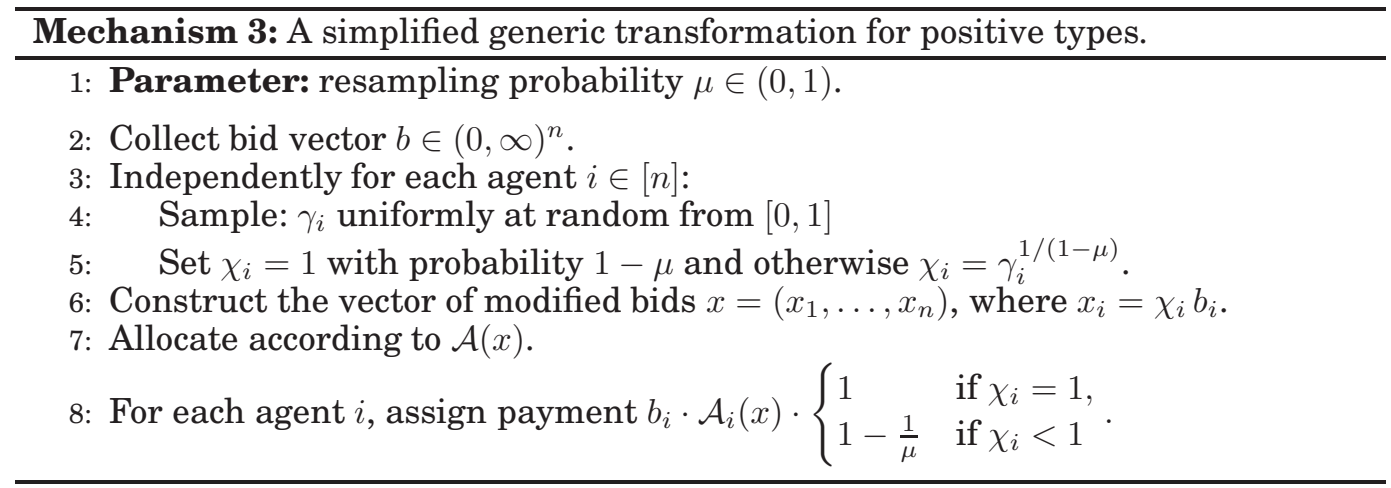

We prove that Mechanism 3 is equivalent to the generic transformation (Mechanism 2) with a canonical self-resampling procedure (Algorithm 1).

Proposition 4.7. The allocation and payments in Mechanism 3 coincide with those in Mechanism 2 with a canonical self-resampling procedure. 
To prove Proposition 4.7, we provide a non-recursive version of the canonical selfresampling procedure (Algorithm 1), which we call ONESHOT. We argue that the output of Mechanism 3 is identical to the output of the mechanism obtained by plugging ONESHOT into Mechanism 27 ONESHOT is also essential for the analysis in Section 5.

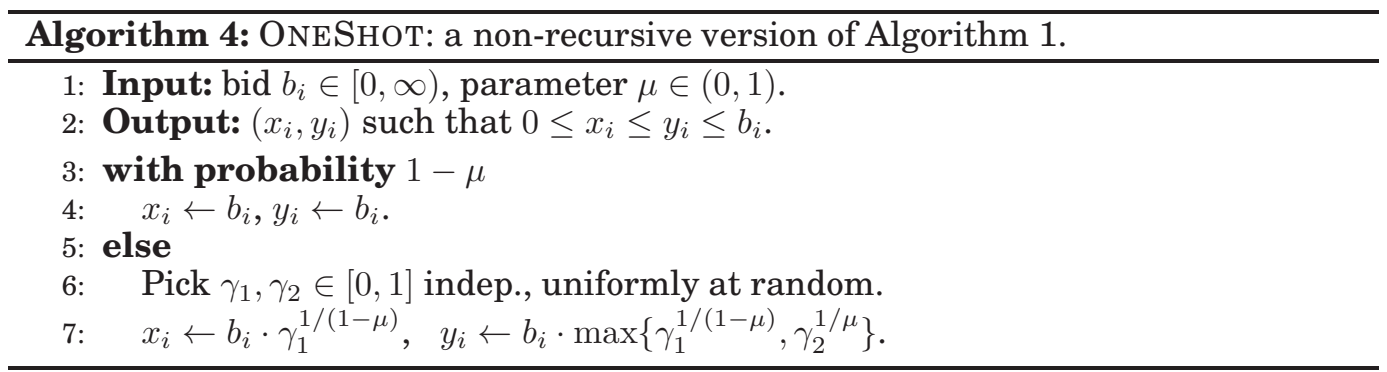

Proposition 4.8. Algorithm 1 and ONESHOT generate the same output distribution: for any bid $b_{i} \in[0, \infty)$, the joint distribution of the pair

$$
\left(x_{i}, y_{i}\right)=\left(x_{i}\left(b_{i} ; w_{i}\right), y_{i}\left(b_{i} ; w_{i}\right)\right)
$$

is the same for both procedures. (Here $w_{i}$ denotes the random seed for each agent $i$.)

The proof of Proposition 4.8 can be found in the Appendix.

Proof of Proposition 4.7, By Proposition 4.8, it suffices to compare Mechanism 3 to Mechanism 2 with self-resampling procedure ONESHOT. To show that the two mechanisms are equivalent, we must show that they yield the same distribution over allocations and the same payments. First we argue about the allocations. In both mechanisms, each bidder's bid $b_{i}$ is independently transformed into a random $x_{i}$, and then the allocation rule $\mathcal{A}$ is applied to the vector $x=\left(x_{1}, \ldots, x_{n}\right)$. Furthermore, the conditional distribution of $x_{i}$ given $b_{i}$ is the same in both cases: $x_{i}=b_{i}$ with probability $1-\mu$, and otherwise $x_{i}=b_{i} \cdot \gamma^{1 /(1-\mu)}$ where $\gamma$ is uniformly distributed in $[0,1]$. Hence, the two mechanisms yield the same distribution over allocations.

To see that the payment rules are the same, consider the distribution function of OnESHOT, as defined in Definition 4.3 .

$$
F\left(a_{i}, b_{i}\right)=\operatorname{Pr}\left[y_{i}\left(b_{i} ; w_{i}\right)<a_{i} \mid y_{i}\left(b_{i} ; w_{i}\right)<b_{i}\right] .
$$

By Proposition 4.8, $F_{i}\left(a_{i}, b_{i}\right)$ is also the distribution function for Algorithm 1. By Proposition 4.4 we have $F\left(a_{i}, b_{i}\right)=a_{i} / b_{i}$, and consequently

$$
F_{i}^{\prime}\left(a_{i}, b_{i}\right) \triangleq \frac{\partial F_{i}\left(a_{i}, b_{i}\right)}{\partial a_{i}}=\frac{1}{b_{i}} .
$$

In particular, neither allocation nor payments in this mechanism depend on the $y_{i}$ 's. Suppressing the $y_{i}$ 's from mechanism $\mathcal{M}$ and plugging in $F_{i}^{\prime}\left(y_{i}, b_{i}\right)=\frac{1}{b_{i}}$, we obtain Mechanism 3. This completes the proof of Proposition 4.7.

${ }^{7}$ This observation is due to [Shnavder et al. 2012]. 


\section{IMPROVED BOUNDS ON WELFARE}

We present improved bounds on the welfare obtained by our generic transformation. We consider two interesting special cases when the agents' private types are, respectively, always positive and always negative. In the second case, agents are contractors who incur costs and get paid by the mechanism; one such example is a shortest paths mechanism considered in Section 6 .

We consider the approximation that is achieved by the mechanism as a function of the approximation of the original allocation rule. Recall that our generic transformation creates a mechanism with an allocation that is identical to the original allocation with probability at least $1-n \mu$. For positive types this immediately implies a bound on the approximation which degrades with $n$, the number of agents. (For negative types such bound does not immediately follow since the cost in the low probability event might be prohibitively high.) For both settings, we present a similar bound that does not degrade with $n$.

\subsection{Positive private types}

Assume that the agents' types are always positive, more specifically that the type space is $\mathcal{T}=(0, \infty)^{n}$. Recall that for agents' types $t \in \mathcal{T}$ the social welfare of an outcome $o$ is defined to $\operatorname{SW}(o, t)=\sum_{i \in N} t_{i} a_{i}(o)$. The optimal social welfare is $\mathrm{OPT}(t)=\max _{o \in O} \operatorname{SW}(o, t)$, where $O$ is the set of all feasible outcomes. (A mechanism with) an allocation rule $\mathcal{A}$ is $\alpha$-approximate if it holds that

$$
\alpha \cdot \mathbb{E}[\operatorname{SW}(\mathcal{A}(t), t)] \geq \operatorname{OPT}(t) \text { for every } t .
$$

THEOREM 5.1. Consider the setting in Theorem $4.5\left(\right.$ c), so that $\mathcal{T}=(0, \infty)^{n}$ and each $f_{i}$ is the canonical self-resampling procedure. If allocation rule $\mathcal{A}$ is $\alpha$-approximate, then mechanism $\operatorname{AllocToMech}(\mathcal{A}, \mu, \mathbf{f})$ is $\alpha /\left(1-\frac{\mu}{2-\mu}\right)$-approximate.

PROOF. Fix a bid vector $b$, and let $o^{*}$ be the corresponding optimal allocation. Recall that our mechanism outputs allocation $\mathcal{A}(x)$, where $x$ is the vector of randomly modified bids. As the original allocation rule $\mathcal{A}$ is $\alpha$-approximate, by Equation (11) it holds that $\alpha \cdot \operatorname{SW}(\mathcal{A}(x), x) \geq \mathrm{OPT}(x)$. We will show that

$$
\mathbb{E}\left[x_{i}\right]=\left(1-\frac{\mu}{2-\mu}\right) b_{i} \text { for each agent } i .
$$

Thus when we evaluate $o^{*}$ with respect to bids $x$ we get:

$$
\begin{aligned}
\alpha \cdot \operatorname{SW}(A(x), x) & \geq \operatorname{OPT}(x) \geq \operatorname{SW}\left(o^{*}, x\right)=\sum_{i \in N} x_{i} a_{i}\left(o^{*}\right) \\
\alpha \cdot \mathbb{E}[\operatorname{SW}(A(x), x)] & =\mathbb{E}\left[\sum_{i \in N} x_{i} a_{i}\left(o^{*}\right)\right] \\
& =\sum_{i \in N}\left(1-\frac{\mu}{2-\mu}\right) b_{i} a_{i}\left(o^{*}\right)=\left(1-\frac{\mu}{2-\mu}\right) \operatorname{OPT}(b) .
\end{aligned}
$$

It remains to prove Equation (12). Let us use ONESHOT to describe the canonical self-resampling procedure. Recall that ONESHOT generates $x_{i}=x_{i}\left(b_{i} ; w_{i}\right)$ by setting $x_{i}=b_{i}$ with probability $1-\mu$, and otherwise sampling $\gamma_{1}$ uniformly at random in $[0,1]$ and outputting $x_{i}=b_{i} \cdot \gamma_{1}^{1 /(1-\mu)}$. Hence

$$
\begin{aligned}
\mathbb{E}\left[x_{i} \mid x_{i}<b_{i}\right] & =\int_{0}^{1} b_{i} \cdot \gamma_{1}^{1 /(1-\mu)} d \gamma_{1}=b_{i} \cdot \frac{1}{1+\frac{1}{1-\mu}}=b_{i} \cdot\left(1-\frac{1}{2-\mu}\right) \\
\mathbb{E}\left[x_{i}\right] & =(1-\mu) \cdot b_{i}+\mu \cdot \mathbb{E}\left[x_{i} \mid x_{i}<b_{i}\right]=b_{i} \cdot\left(1-\frac{\mu}{2-\mu}\right) .
\end{aligned}
$$


For arbitrary self-resampling procedures $f_{i}$ with support $\mathbb{R}_{+}$, Equation (12) can be replaced by $\mathbb{E}\left[x_{i}\right] \geq(1-\mu) b_{i}$, which gives a slightly weaker result, namely an $\frac{\alpha}{1-\mu}$ approximation to the social welfare.

\subsection{Negative private types}

Now assume that the agents' types are always negative, more specifically that $\mathcal{T}=$ $(-\infty, 0)^{n}$. For negative types approximation is defined with respect to the social cost, which is the negation of the social welfare. An algorithm is $\alpha$-approximate if for every input it outputs an outcome with cost at most $\alpha$ times the optimal cost. We present an approximation bound for an $h$-canonical self-resampling procedure, for a suitably chosen $h$.

TheORem 5.2. Consider the setting in Theorem 4.5 Assume that $\mathcal{T}=(-\infty, 0)^{n}$ and that each $f_{i}$ is the $h$-canonical self-resampling procedure, where $h\left(z_{i}, b_{i}\right)=$ $b_{i} / \sqrt{z_{i}}$. Suppose $\mu \in\left(0, \frac{1}{2}\right)$. If allocation rule $\mathcal{A}$ is $\alpha$-approximate, then mechanism $\operatorname{AllocToMech}(\mathcal{A}, \mu, \mathbf{f})$ is $\alpha\left(1+\frac{\mu}{1-2 \mu}\right)$-approximate.

The proof of this theorem is almost identical to that of Theorem 5.1 , and thus is omitted. The main modification is that Equation (12) is replaced by the following lemma:

LEMMA 5.3. In the setting of Theorem 5.2 letting $x^{h}$ be the vector of modified types, it holds that

$$
\mathbb{E}\left[x_{i}^{h}\right]=b_{i}\left(1+\frac{\mu}{1-2 \mu}\right) \text { for all } i .
$$

Proof. Recall that $x^{h}$ is defined by Equation (9). As in the proof of Equation (12), we will use ONESHOT to describe the canonical self-resampling procedure. It follows that

$$
\begin{aligned}
\mathbb{E}\left[x_{i}^{h} \mid x_{i}<b_{i}\right] & =\int_{0}^{1} \frac{b_{i}}{\sqrt{\gamma_{1}^{\frac{1}{(1-\mu)}}}} d \gamma_{1}=\int_{0}^{1} b_{i} \cdot \gamma_{1}^{-\frac{1}{2(1-\mu)}} d \gamma_{1}=b_{i} \cdot \frac{1}{1-\frac{1}{2(1-\mu)}}=b_{i} \cdot\left(1+\frac{1}{1-2 \mu}\right), \\
\mathbb{E}\left[x_{i}^{h}\right] & =(1-\mu) \cdot b_{i}+\mu \cdot \mathbb{E}\left[x_{i}^{h} \mid x_{i}^{h}<b_{i}\right]=b_{i} \cdot\left(1+\frac{\mu}{1-2 \mu}\right) .
\end{aligned}
$$

\section{APPLICATIONS TO OFFLINE MECHANISM DESIGN}

The VCG mechanism for shortest paths. The seminal paper Nisan and Ronen [2001] has presented the following question: is there a computational overhead in computing payments that will induce agents to be truthful, compared to the computation burden of computing the allocation. One of their examples is the VCG mechanism for the shortest path mechanism design problem, where a naive computation of VCG payments requires additional computation of $n$ shortest path instances. Yet, an explicit payment computation is not the real goal, it is just a means to an end. The real goal is inducing the right incentives. Our procedure shows that without any overhead in computation, if we move to a randomized allocation rule and settle for truthfulness in expectation (and a small loss in performance) one can induce the right incentives.

The shortest path mechanism design problem is the following. We are given a graph $G=(V, E)$ and a pair of source-target nodes $\left(v_{s}, v_{t}\right)$. Each agent $e$ controls an edge $e \in E$ and has a cost $c_{e}>0$ if picked (thus $v_{e}=-c_{e}<0$ and $\mathcal{T}_{e}=(-\infty, 0)$ for every $e$ ). That cost is private information, known only to agent $e$. The mechanism designer's goal is to pick a path $P$ from node $v_{s}$ to node $v_{t}$ in the graph with minimal total cost, 
that is $\sum_{e \in P} c_{e}$ is minimal. Assume that there is no edge that forms a cut between $v_{s}$ and $v_{t}$.

The VCG mechanism is an cost-optimal and truthful mechanism for this problem. It computes a shortest path $P$ with respect to the reported costs and pays to an agent $e$ the difference between the cost of the shortest path that does not contains $e$ and the total cost shortest path excluding the cost of $e$. A naive implementation of the VCG mechanism requires computing $|P|+1$ shortest path instances (where $|P|$ denotes the number of edges in path $P$ ). VCG is deterministic, truthful and cost-optimal.

Let EFF an the cost-optimal allocation rule for the shortest path problem. We can use our general procedure to derive the following result (its proof follows directly from Theorem 4.5 and Theorem 5.2.

THEOREM 6.1. Fix any $\mu \in\left(0, \frac{1}{2}\right)$. For each agent $i$, let $f_{i}$ be the $h$-canonical selfresampling procedure, where $h\left(z_{i}, b_{i}\right)=b_{i} / \sqrt{z_{i}}$. Let $\mathcal{M}=\operatorname{AllocToMech}\left(\mathrm{EFF}, \mu,\left\{f_{i}\right\}\right)$ be the mechanism created by applying AllocToMech() to EFF. Then $\mathcal{M}$ has the following properties:

- It is truthful and universally individually rational.

- It only computes one shortest paths instance.

- It outputs a path with expected length at most $\left(1+\frac{\mu}{1-2 \mu}\right)$ times the length of the shortest path.

Recall that parameter $\mu$ controls the trade-off between approximation ratio and the rebate size $R_{i}$, which for a given random seed is proportional to $\frac{1}{\mu}$.

Communication overhead of payment computation. Babaioff et al. [2013] show that there exists a monotone deterministic allocation rule for which the communication required for computing the allocation is factor $\Omega(n)$ less than the communication required to computing prices. This implies that inducing the correct incentives deterministically has a large overhead in communication. Assume that instead of requiring explicit computation of payments we are satisfied with inducing the correct incentives using a randomized mechanism. In such case our reduction shows that the deterministic lower bound cannot be extended to randomized mechanisms, if we allow a small error in the allocation.

More concretely, consider a single parameter domain with types that are positive, $\mathcal{T}_{i}=(0, \infty)$ (as in [Babaioff et al. 2013]). For all $i$, use the canonical self-resampling procedure. Consider any monotone allocation rule $\mathcal{A}$. We can apply Theorem 4.5 to obtain a randomized mechanism that is truthful and only executes that allocation rule $\mathcal{A}$ once (thus has no communication overhead at all) and has exactly the same allocation with probability at least $(1-\mu)^{n}$. For any $\epsilon>0$ we can find $\mu>0$ such that the error probability is less than $\epsilon$.

\section{MULTI-ARMED BANDIT MECHANISMS}

In this section we apply the main result to multi-armed bandit (MAB) mechanisms: single-parameter mechanisms in which the allocation rule is (essentially) an MAB algorithm parameterized by the bids. As in any single-parameter mechanism, agents submit their bids, then the allocation rule is run, and then the payments are assigned. This application showcases the full power of the main result, since in the MAB setting the allocation rule is only run once, and (in general) cannot be simulated as a computational routine without actually implementing the allocation.

Focusing on the stochastic setting, we design truthful MAB mechanisms with the same regret guarantees as the best MAB algorithms such as UCB1 [Auer et al. 2002a]. First, we prove that allocation rules derived from UCB1 and similar MAB algorithms 
are in fact monotone, and hence give rise to truthful MAB mechanisms. Second, we provide a new allocation rule with the same regret guarantees that is ex-post monotone, and hence gives rise to an ex-post truthful MAB mechanism. Third, we use this new allocation rule to obtain an unconditional separation between the power of randomized and deterministic ex-post truthful MAB mechanisms.

\subsection{Preliminaries: MAB mechanisms}

An MAB mechanism [Babaioff et al. 2014; Devanur and Kakade 2009] operates as follows. There are $n$ agents. Each agent $i$ has a private value $v_{i}$ and submits a bid $b_{i}$. We assume that $b_{i}, v_{i} \in\left[0, b_{\max }\right]$, where $b_{\max }$ is known a priori. The allocation consists of $T$ rounds, where $T$ is the time horizon. In each round $t$ the allocation rule chooses one of the agents, call it $i=i(t)$, and observes a click reward $\pi(t) \in[0,1]$ for this choice; the chosen agent $i$ receives $v_{i} \pi(t)$ units of utility. Payments are assigned after the last round of the allocation. Note that the social welfare of the mechanism is equal to the total value-adjusted click reward: $\sum_{t=1}^{T} v_{i(t)} \pi(t)$.

The special case of $0-1$ click rewards corresponds to the scenario in which agents are advertisers in a pay-per-click auction, and choosing agent $i$ in a given round $t$ means showing this agent's ad. Then the click reward $\pi(t)$ is the click bit: 1 if the ad has been clicked, and 0 otherwise. Following the web advertising terminology, we will say that in each round, an impression is allocated to one of the agents.

Formally, an $M A B$ allocation rule $\mathcal{A}$ is an online algorithm parameterized by $n, T, b_{\max }$ and the bids $b$. In each round it allocates the impression and observes the click reward. Absent truthfulness constraints, the objective is to maximize the reported welfare: $\sum_{t=1}^{T} b_{i(t)} \pi(t)$. This formulation generalizes MAB algorithms: the latter are precisely MAB allocation rules with all bids set to 1 .

Given an MAB algorithm $\hat{\mathcal{A}}$, there is a natural way to transform it into an MAB allocation rule $\mathcal{A}$. Namely, $\mathcal{A}$ runs algorithm $\hat{\mathcal{A}}$ with modified click rewards: if agent $i$ is chosen in round $t$ then the click reward reported to $\hat{\mathcal{A}}$ is $\hat{\pi}(t)=\left(b_{i} / b_{\max }\right) \pi(t)$. We will say that algorithm $\hat{\mathcal{A}}$ induces allocation rule $\mathcal{A}$. From now on we will identify an $\mathrm{MAB}$ algorithm with the induced allocation rule, e.g. allocation rule UCB1 is induced by algorithm UCB1 [Auer et al. 2002a].

We will focus on the stochastic MAB setting: in all rounds $t$ in which an agent $i$ is chosen, the click reward $\pi(t)$ is an independent random sample from some fixed distribution on $[0,1]$ with expectation $\mu_{i}:$ Following the web advertisement terminology, we will call $\mu_{i}$ the click-through rate (CTR) of agent $i$. The CTRs are fixed, but no further information about them (such as priors) is revealed to the mechanism.

Regret. The performance of an MAB allocation rule is quantified in terms of regret:

$$
R(T ; b ; \mu) \triangleq T \max _{i}\left[b_{i} \mu_{i}\right]-\mathbb{E}\left[\sum_{t=1}^{T} b_{i(t)} \mu_{i(t)}\right],
$$

the difference in expected click rewards between the algorithm and the benchmark: the best agent in hindsight, knowing the $\mu_{i}$ 's. We focus on $R(T) \triangleq \max R(T ; b ; \mu)$, where the maximum is taken over all CTR vectors $\mu$ and all bid vectors $b$ such that $b_{i} \leq 1$ for all i. 9

Regret guarantees from the vast literature on MAB algorithms easily translate to MAB allocation rules. In particular, allocation rule UCB1 has regret $R(T)=O(\sqrt{n T \log T})$ [Auer et al. 2002a], which is nearly matching the information-

\footnotetext{
${ }^{8}$ The exact shape of this distribution is not essential. E.g. in the advertising example $\pi(t) \in\{0,1\}$.

${ }^{9}$ We define $R(T)$ with $b_{\max }=1$ merely to simplify the notation. All regret bounds (scaled up by a factor of $b_{\max }$ ) hold for an arbitrary $b_{\max }$.
} 
theoretically optimal regret bound $\Theta(\sqrt{n T})$ Auer et al. 2002b; Audibert and Bubeck 2010]. The stochastic MAB setting tends to be easier if the best agent is much better than the second-best one. Let us sort the agents so that $b_{1} \mu_{1} \geq b_{2} \mu_{2} \geq \ldots \geq b_{n} \mu_{n}$. The gap $\delta$ of the problem instance is defined as $\left(b_{1} \mu_{1}-b_{2} \mu_{2}\right) / b_{\max }$. The $\delta$-gap regret $R_{\delta}(T)$ is defined as the worst-case regret over all problem instances with gap $\delta$. Allocation rule UCB1 achieves $R_{\delta}(T)=O\left(\frac{n}{\delta} \log T\right)$ [Auer et al. 2002a]; there is a lower bound $R_{\delta}(T)=\Omega\left(\min \left(\frac{n}{\delta} \log T, \sqrt{n T}\right)\right)$ Lai and Robbins 1985; Auer et al. 2002b; Kleinberg et al. 2008a].

Click realizations. A click realization is a $n \times T$ table $\rho$ in which the $(i, t)$ entry $\rho_{i}(t)$ is the click reward (e.g., the click bit) that agent $i$ receives if it is played in round $t$. Note that in order to fully define the behavior of any algorithm on all bid vectors one may need to specify all entries in the table, whereas only a subset thereof is revealed in any given run. We view $\rho$ as a realization of nature's random seed. Thus, we can now define ex-post truthfulness and other ex-post properties: informally, ex-post property is a property that holds for every given click realization.

For each agent $i$, round $t$, bid vector $b$ and click realization $\rho$, let $\mathcal{A}_{i}^{t}(b ; \rho)$ denote the probability that MAB allocation rule $\mathcal{A}$ allocates the impression at round $t$ to agent $i$. (If $\hat{\mathcal{A}}$ is deterministic, the probability $\hat{\mathcal{A}}_{i}^{t}(\rho)$ is trivial: either 0 or 1.)

For MAB algorithm $\hat{\mathcal{A}}$, define $\hat{\mathcal{A}}_{i}^{t}(\rho)$ similarly.

\subsection{Truthfulness and monotonicity}

Theorem 4.5 (c) reduces the problem of designing truthful MAB mechanisms to that of designing monotone $\mathrm{MAB}$ allocations. Let us state this reduction explicitly:

THEOREM 7.1. Consider the stochastic MAB mechanism design problem. Let $\mathcal{A}$ be a stochastically monotone (resp., ex-post monotone) MAB allocation rule. Applying the transformation in Theorem $4.5 \mathrm{c} 10$ to $\mathcal{A}$ with parameter $\mu$, we obtain a mechanism $\mathcal{M}$ such that:

(a) $\mathcal{M}$ is stochastically truthful (resp., ex-post truthful), ex-post no-positivetransfers, and universally ex-post individually rational.

(b) for each click realization, the difference in expected welfare between $\mathcal{A}$ and $\mathcal{M}$ is at $\operatorname{most} \mu n T b_{\max }$.

Note that the theorem provides two distinct types of guarantees: game-theoretic guarantees in part (a), and performance guarantees in part (b).

We show that a very general class of deterministic MAB algorithms induces monotone MAB allocation rules (to which Theorem 7.1 can be applied).

Definition 7.2. In a given run of an MAB algorithm, the round-t statistics is a pair of vectors $(\pi, \nu)$, where the $i$-th component of $\pi$ (resp., $\nu$ ) is equal to the total payoff (resp., the number of impressions) of agent $i$ in rounds 1 to $t-1$, for each agent $i$. Vectors $\pi$ and $\nu$ are called $p$-stats vector and $i$-stats vector, respectively.

Definition 7.3. A deterministic MAB algorithm $\hat{\mathcal{A}}$ is called well-formed if for each round $t$ and agent $i$, letting $(\pi, \nu)$ be the round- $t$ statistics, the following properties hold:

- $\left[\hat{\mathcal{A}}_{i}^{t}(\rho)\right.$ is determined by $\left.(\pi, \nu)\right]$ there is a function $\chi_{i}(\pi ; \nu)$ that depends only on the round- $t$ statistics such that $\hat{\mathcal{A}}_{i}^{t}(\rho)=\chi_{i}(\pi ; \nu)$ for any click realization $\rho$ and all $t$.

- [ $\chi$-monotonicity $] \quad \chi_{i}(\pi ; \nu)$ is non-decreasing in $\pi_{i}$ for any fixed $\left(\pi_{-i}, \nu\right)$.

\footnotetext{
${ }^{10}$ Theorem 4.5 c) is stated for the type space $T=(0, \infty)^{n}$, but it trivially extends to the case $T=\left(0, b_{\max }\right)^{n}$.
} 
- [ $\chi$-IIA $]$ for each round $t$, any three distinct agents $\{i, j, l\}$ and any fixed $\left(\pi_{-i}, \nu_{-i}\right)$, changing $\left(\pi_{i}, \nu_{i}\right)$ cannot transfer an impression from $j$ to $l$.

The $\chi$-IIA property above is reminiscent of Independence of Irrelevant Alternatives (IIA) property in the Social Choice literature (hence the name). A similar but technically different property is essential in the analysis of deterministic MAB allocation rules in [Babaioff et al. 2014].

Remark 7.4. For a concrete example of a well-formed MAB algorithm, consider (a version of) UCB1 11 The algorithm is very simple: in each round $t$, it chooses agent

$$
\min \left(\arg \max _{i}\left(\pi_{i}(t) / \nu_{i}(t)+\sqrt{8 \log (T) / \nu_{i}(t)}\right)\right) .
$$

LEMMA 7.5. In the stochastic MAB mechanism design problem, let $\mathcal{A}$ be a $M A B$ allocation rule induced by a well-formed $M A B$ algorithm. Then $\mathcal{A}$ is stochastically monotone.

PROOF. We will use an alternative way to define a realization of random click rewards: a stack-realization is a $n \times T$ table in which the $(i, t)$ entry is the click bit that agent $i$ receives the $t$-th time she is played. Clearly a stack-realization and a bid vector uniquely determine the behavior of $\mathcal{A}$. We will show that:

$\mathcal{A}$ is monotone for each stack-realization.

Then $\mathcal{A}$ is monotone in expectation over any distribution over stack-realizations, and in particular it is monotone in expectation over the random clicks in the stochastic MAB setting, so the Lemma follows.

Let us prove Claim (13). Throughout the proof, fix stack-realization $\sigma$, agent $i$, and bid vector $b_{-i}$. Consider two bids $b_{i}<b_{i}^{+}$. The claim asserts that agent $i$ receives at least as many clicks with bid $b_{i}^{+}$than with bid $b_{i}$.

Let us introduce some notation (letting $b_{i}$ be the bid of agent $i$ ). Let $\mathcal{A}\left(b_{i}, t\right)$ be the agent selected by the allocation rule in round $t$. For each agent $j$, let $\nu_{j}\left(b_{i}, t\right)$ and $\pi_{j}\left(b_{i}, t\right)$ be, respectively, the total number of impressions and the total click reward of agent $j$ in the first $t$ rounds. Let $\hat{\pi}_{j}\left(b_{i}, t\right)=\left(b_{j} / b_{\max }\right) \pi_{j}\left(b_{i}, t\right)$ be the corresponding total modified click reward. Let $\nu\left(b_{i}, t\right)$ (resp., $\pi\left(b_{i}, t\right)$ and $\hat{\pi}\left(b_{i}, t\right)$ ) be the $n$-dimensional vector whose $j$-th component is $\nu_{j}\left(b_{i}, t\right)$ (resp., $\pi_{j}\left(b_{i}, t\right)$ and $\hat{\pi}_{j}\left(b_{i}, t\right)$ ) for each agent $j$.

Note that $\left(\hat{\pi}\left(b_{i}, t\right), \nu\left(b_{i}, t\right)\right)$ is the round- $t$ statistics for the MAB algorithm that $\mathcal{A}$ is induced by. For each agent $j, \nu_{j}\left(b_{i}, t\right)$ uniquely determines $\pi_{j}\left(b_{i}, t\right)$ :

$$
\pi_{j}\left(b_{i}, t\right)=\sum_{s=1}^{\nu_{j}} \sigma(j, s) \quad \text { where } \nu_{j}=\nu_{j}\left(b_{i}, t\right) .
$$

Let us overview the forthcoming technical argument. We will show by induction on $t$ that $\nu_{i}\left(b_{i}, t\right) \leq \nu_{i}\left(b_{i}^{+}, t\right)$ for all $t$. For the induction step we only need to worry about the case when the claim holds for a given $t$ with equality. In this case we show that $\nu_{-i}\left(b_{i}, t\right)=\nu_{-i}\left(b_{i}^{+}, t\right)$. This is trivial for $n=2$ agents; the general case requires a rather delicate argument that uses the $\chi$-IIA property in Definition 7.3 [12]

Now let us carry out the proofs in detail. First, denote $\nu_{*}\left(b_{i}, t\right) \triangleq t-\nu_{i}\left(b_{i}, t\right)$, and let us show that for any two rounds $t, s$ it holds that

$$
\nu_{*}\left(b_{i}, t\right)=\nu_{*}\left(b_{i}^{+}, s\right) \Rightarrow \nu_{-i}\left(b_{i}, t\right)=\nu_{-i}\left(b_{i}^{+}, s\right) .
$$

\footnotetext{
${ }^{11}$ To ensure the $\chi$-IIA property, we use a slightly modified version of UCB1: $\log T$ is used instead of $\log t$, and min is used to break ties (instead of an arbitrary rule). This change does not affect regret guarantees. We will denote this version as UCB1 without further notice.

${ }^{12}$ Also, we will use the fact that the probabilities $\chi_{j}(\hat{\pi}, \nu)$ in Definition7.3 do not depend on the round (given $j$ and $(\hat{\pi}, \nu)$ ). This is the only place in any of the proofs where we invoke this fact.
} 
Let us use induction on $\nu_{*}\left(b_{i}, t\right)$. For $\nu_{*}\left(b_{i}, t\right)=0$ the statement is trivial. For the induction step, suppose Equation (15) holds whenever $\nu_{*}\left(b_{i}, t\right)=\nu_{*}$, and let us suppose $\nu_{*}\left(b_{i}, t\right)=\nu_{*}\left(b_{i}^{+}, s\right)=\nu_{*}+1$. Let $t^{\prime}$ and $s^{\prime}$ be the latest rounds such that $\nu_{*}\left(b_{i}, t^{\prime}\right)=\nu_{*}\left(b_{i}^{+}, s^{\prime}\right)=\nu_{*}$. By the induction hypothesis, $\nu_{-i}\left(b_{i}, t^{\prime}\right)=\nu_{-i}\left(b_{i}^{+}, s^{\prime}\right)$. It remains to prove that $\mathcal{A}\left(b_{i}, t^{\prime}+1\right)=\mathcal{A}\left(b_{i}^{+}, s^{\prime}+1\right)$, i.e. that the allocation rule's selections in round $t^{\prime}+1$ given bids $\left(b_{-i}, b_{i}\right)$, and in round $s^{\prime}+1$ given bids $\left(b_{-i}, b_{i}^{+}\right)$, are the same 13 By Definition 7.3 these selections are uniquely determined (given the stackrealization) by the bids and the impression counts $\nu$. By the choice of $t^{\prime}$ and $s^{\prime}$, neither of the two selections is $i$, so by the $\chi$-IIA condition in Definition 7.3 the selections are uniquely determined by $b_{-i}$ and $\nu_{-i}$, and hence are the same. This proves Equation (15).

Now, to prove Claim (13) it suffices to show that for all $t$

$$
\nu_{i}\left(b_{i}, t\right) \leq \nu_{i}\left(b_{i}^{+}, t\right) .
$$

Let us use induction on $t$. The claim is trivial for $t=1$, since the impression of agent $i$ in round 1 does not depend on $(b ; \sigma)$. For the induction step, assume that the assertion Equation (16) holds for some $t$, and let us prove it for $t+1$. Note that (using the notation from Definition 7.3 .

$$
\nu_{i}\left(b_{i}, t+1\right)=\nu_{i}\left(b_{i}, t\right)+\chi_{i}\left(\hat{\pi}\left(b_{i}, t\right) ; \nu\left(b_{i}, t\right)\right) .
$$

Now, $\nu_{i}\left(b_{i}, t\right) \leq \nu_{i}\left(b_{i}^{+}, t\right)$ by induction hypothesis. If the inequality is strict then Equation (16) trivially holds for $t+1$. Now suppose $\nu_{i}\left(b_{i}, t\right)=\nu_{i}\left(b_{i}^{+}, t\right)$. Then by Equation (15) we have $\nu\left(b_{i}, t\right)=\nu\left(b_{i}^{+}, t\right)$. Moreover, by Equation (14) we have $\pi\left(b_{i}, t\right)=\pi\left(b_{i}^{+}, t\right)$ and therefore $\hat{\pi}_{-i}\left(b_{i}, t\right)=\hat{\pi}_{-i}\left(b_{i}^{+}, t\right)$ and $\hat{\pi}_{i}\left(b_{i}, t\right)<\hat{\pi}_{i}\left(b_{i}^{+}, t\right)$. Thus, by the $\chi$-monotonicity property in Definition 7.3 we have

$$
\chi_{i}\left(\hat{\pi}\left(b_{i}, t\right) ; \nu\left(b_{i}, t\right)\right) \leq \chi_{i}\left(\hat{\pi}\left(b_{i}^{+}, t\right) ; \nu\left(b_{i}^{+}, t\right)\right) .
$$

This concludes the proof of Equation (16), and that Claim (13).

\subsection{Truthfulness and regret}

In this subsection we focus on the stochastic MAB setting, and consider the trade-off between regret and various notions of truthfulness. Ideally, one would like an MAB mechanism to be truthful in the strongest possible sense (universally ex-post), and have the same regret bounds as optimal MAB algorithms.

Let us start with some background. In Babaioff, Sharma and Slivkins [Babaioff et al. 2014 it was proved that any deterministic mechanism that is ex-post truthful and ex-post normalized (under very mild restrictions), and any distribution over such deterministic mechanisms, incurs much higher regret than an optimal MAB algorithm such as UCB1. Namely, the lower bound in [Babaioff et al. 2014] states that $R(T)=\Omega\left(n^{1 / 3} T^{2 / 3}\right)$, whereas UCB1 has regret $R(T)=O(\sqrt{n T \log T})$. 14 For $\delta$-gap instances the difference is even more pronounced: the analysis in [Babaioff et al. 2014] provides a polynomial lower bound of $R_{\delta}(T)=\Omega\left(\delta T^{\lambda}\right)$ for some $\lambda>0$, whereas UCB1 achieves logarithmic regret $R_{\delta}(T)=O\left(\frac{n}{\delta} \log T\right)$.

Our first result is that we can use the machinery from Section 7.2 to match the regret of UCB1 for truthful mechanisms. We apply Theorem 7.1 (with $\mu=\frac{1}{T}$ ) and Lemma[7.5 to UCB1 to obtain the following corollary:

\footnotetext{
${ }^{13}$ Then $\nu_{-i}\left(b_{i}, t\right)=\nu_{-i}\left(b_{i}^{+}, s\right)$ because in all rounds from $t^{\prime}+2$ to $t$ (resp., from $s^{\prime}+2$ to $s$ ) agent $i$ is played. ${ }^{14}$ Following the literature on regret minimization, we are mainly interested in the asymptotic behavior of $R(T)$ as a function of $T$ when $n$ is fixed.
} 
COROLLARY 7.6. In the stochastic MAB mechanism design problem, there exists a mechanism $\mathcal{M}$ such that

(a) $\mathcal{M}$ is stochastically truthful, ex-post no-positive-transfers, universally ex-post individually rational.

(b) $\mathcal{M}$ has regret $R(T)=O(\sqrt{n T \log T})$ and $\delta$-gap regret $R_{\delta}(T)=O\left(\frac{n}{\delta} \log T\right)$.

Remark 7.7. The regret and $\delta$-gap regret in the above theorem are within small factors (resp., $O(\sqrt{\log T})$ and $O(1))$ of the best possible for any MAB allocation rule.

Remark 7.8. [Babaioff et al. 2014 provides a weaker result which transforms any monotone MAB algorithm such as UCB1 into a truthful and normalized MAB mechanism with matching regret bounds. The guarantees in [Babaioff et al.2014] are weaker for the following reasons. First, it only applies to 0-1 click rewards, whereas our setting allows for arbitrary click rewards in $[0,1]$. Second, the individual rationality guarantee in [Babaioff et al. 2014] is much weaker: an agent may be charged more than her bid (which never happens in our mechanism), and the charge may be huge, as high as $b_{i} \times(4 n)^{T}$; thus, a risk-averse agent may be reluctant to participate. Third, the nopositive-transfers guarantee is weaker: for some realizations of the click rewards the expected payment may be negative. Finally, the payment rule in [Babaioff et al. 2014] requires (as stated) a prohibitively expensive computation.

The truthfulness in Corollary 7.6 is only in expectation over the random click rewards. Thus, after seeing a specific realization of the rewards an agent might regret having been truthful. Accordingly, we would like a stronger property: ex-post truthfulness, i.e. truthfulness for every given realization of the rewards.

The main result of this section is an ex-post truthful MAB mechanism with optimal regret bounds. Unlike Corollary 7.6, this result requires designing a new MAB allocation rule 15 This allocation rule and its analysis are the main technical contributions.

THEOREM 7.9. In the stochastic MAB mechanism design problem, there is a mechanism $\mathcal{M}$ such that

(a) $\mathcal{M}$ is ex-post truthful, ex-post no-positive-transfers, and universally ex-post individually rational.

(b) $\mathcal{M}$ has regret $R(T)=O(\sqrt{n T \log T})$ and $\delta$-gap regret $R_{\delta}(T)=O\left(\frac{n}{\delta} \log T\right)$.

The theorem follows from Theorem 7.1 (with $\mu=\frac{1}{\text { ) }}$ ) if there exists an MAB allocation rule that is ex-post monotone and has the claimed regret bounds. Below we provide such allocation rule, called NewCB.

LEMMA 7.10. NewCB is ex-post monotone and satisfies the regret bounds in Theorem 7.9 (b).

Remark 7.11. NewCB is deterministic. While not essential for Theorem7.9, this fact confirms the intuition from [Babaioff et al. 2014] that the main obstacle for deterministic ex-post truthful MAB mechanisms is insufficient observable information to compute payments rather than ex-post monotonicity of an allocation rule.

NewCB maintains a set of active agents; initially all agents are active. For each round $t$, there is a designated agent $i=1+(t \bmod n)$. If this agent is active, then it is allo-

\footnotetext{
${ }^{15}$ In particular, the allocation rule induced by UCB1 is not ex-post monotone and thus cannot be used to achieve ex-post truthfulness using the results of Section 7.2 To see that, consider a simple setting with two agents and two rounds, and a click realization in which both agents are not clicked at the first round, but are clicked at the second. With this click realization, an agent might be better off decreasing his bid in order to lose (i.e., not be selected in) the first round, and then win (i.e., be selected in) the second round.
} 
cated. Else, an active agent is chosen (according to some fixed ordering on the agents) and allocated. For each agent $i$, lower and upper confidence bounds $\left(L_{i}, U_{i}\right)$ on the product $b_{i} \mu_{i}$ are maintained (recall that $\mu_{i}$ is the CTR of agent $i$ ). After each round, each agent is de-activated if its upper confidence bound is smaller than someone else's lower confidence bound. The pseudocode is in Algorithm 5 .

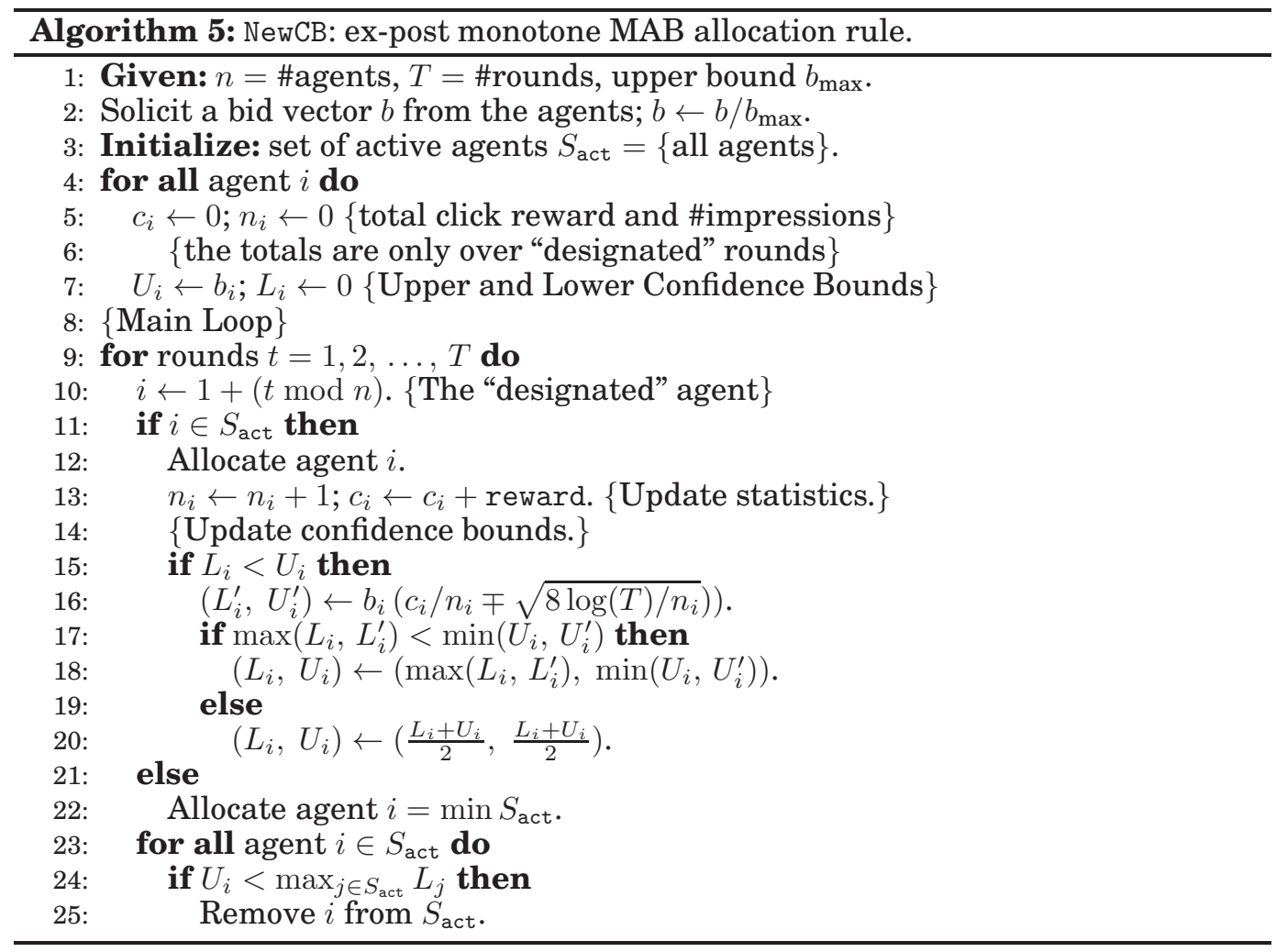

Fix realization $\rho$ and bid vector $b$. Let $S_{\text {act }}(t, b)$ be the set of active agents in the beginning of round $t$. For each agent $i$, let $L_{i}(t, b)$ and $U_{i}(t, b)$ be the values of $L_{i}$ and $U_{i}$ in the end of round $t$.

The goal of the specific update rules for the confidence bounds (lines 15-20) and the statistics (lines 13) is to guarantee the following two properties:

- the statistics are kept only for rounds when a designated agent is played. Moreover, for each agent $i$ and round $t$, and any two bid vectors $b$ and $b^{\prime}$ we have

$$
\text { if } i \in S_{\text {act }}(t, b) \cap S_{\text {act }}\left(t, b^{\prime}\right) \text { then }\left\{\begin{array}{l}
L_{i}(t, b) / b_{i}=L_{i}\left(t, b^{\prime}\right) / b_{i}^{\prime} \\
U_{i}(t, b) / b_{i}=U_{i}\left(t, b^{\prime}\right) / b_{i}^{\prime}
\end{array} .\right.
$$

- for any fixed realization $\rho$ and bid vector $b$, and each agent $i: L_{i} \leq U_{i}$, and from round to round $L_{i}$ is non-decreasing and $U_{i}$ is non-increasing. In other words, for each round $t$ it holds that

$$
L_{i}(t-1, b) \leq L_{i}(t, b) \leq U_{i}(t, b) \leq U_{i}(t-1, b) .
$$


The ex-post monotonicity follows from these two properties and the de-activation rule (lines 24-25).

Ex-post monotonicity. Let $L^{*}(t, b) \triangleq \max _{i \in S_{\text {act }}(t, b)} L_{i}(t, b)$. Fix agent $i$ and $b_{i}^{+}>b_{i}$, and let $b^{+}=\left(b_{-i}, b_{i}^{+}\right)$be the "alternative" bid vector. Let $\lambda=b_{i} / b_{i}^{+}$.

ClaIM 7.12. We establish the following sequence of claims:

(C1) $L^{*}(t, b)$ is non-decreasing in $t$, for any fixed $b$.

(C2) For each round $t, L^{*}(t, b) \leq \lambda L^{*}\left(t, b^{+}\right)$.

(C3) For each round $t, S_{\text {act }}\left(t, b^{+}\right) \backslash\{i\} \subset S_{\text {act }}(t, b) \backslash\{i\}$.

(C4) In each round $t$ : if $i \in S_{\text {act }}(t, b)$ then $i \in S_{\text {act }}\left(t, b^{+}\right)$.

PROOF. Let us prove the parts (C1-C4) one by one.

(C1). We use property (18) and the de-activation rule. Throughout the proof, we omit the bid vector $b$ from the notation. Fix round $t \geq 2$. Let $i \in S_{\text {act }}(t-1)$ be an agent such that $L^{*}(t-1)=L_{i}(t-1)$. If $i \in S_{\text {act }}(t)$ then

$$
L^{*}(t-1)=L_{i}(t-1) \leq L_{i}(t) \leq L^{*}(t)
$$

Else $i$ is de-activated in round $t$, so

$$
L^{*}(t-1)=L_{i}(t-1) \leq L_{i}(t) \leq U_{i}(t)<L^{*}(t) .
$$

(C2). Suppose, for the sake of contradiction, that $L^{*}(t, b)>\lambda L^{*}\left(t, b^{+}\right)$. Let $j \in S_{\text {act }}(t, b)$ be an agent such that $L_{j}(t, b)=L^{*}(t, b)$. If $j \in S_{\text {act }}\left(t, b^{+}\right)$then by property (17) we have

$$
L^{*}(t, b)=L_{j}(t, b)=\lambda L_{j}\left(t, b^{*}\right) \leq \lambda L^{*}\left(t, b^{+}\right),
$$

contradiction. We conclude that $j \notin S_{\text {act }}\left(t, b^{+}\right)$. Thus with bid vector $b^{+}$agent $j$ gets disqualified during some round $s<t$. Thus,

$$
U_{j}\left(s, b^{+}\right)<L^{*}\left(s, b^{+}\right) \leq L^{*}\left(t, b^{+}\right),
$$

where the second inequality is by Part (C1). Now using property (18) and property (17) (for the right-most inequality), we get that

$$
L^{*}(t, b)=L_{j}(t, b) \leq U_{j}(t, b) \leq U_{j}(s, b)=\lambda U_{j}\left(s, b^{+}\right) .
$$

Thus, $L^{*}(t, b) \leq \lambda L^{*}\left(t, b^{+}\right)$by Equation (19), the desired contradiction.

(C3). Use induction on $t$. The claim trivially holds for $t=1$. Assuming the claim holds for some $t$ we prove it holds for $t+1$. Fix agent $j \in S_{\text {act }}\left(t+1, b^{+}\right) \backslash\{i\}$. We need to prove that $j \in S_{\text {act }}(t+1, b)$.

Note that $j \in S_{\text {act }}\left(t, b^{+}\right)$, and so $j \in S_{\text {act }}(t, b)$ by the induction hypothesis. Therefore

$$
\begin{array}{rlr}
L^{*}(t, b) & \leq \lambda L^{*}\left(t, b^{+}\right) & \text {(by Part }(\mathrm{C} 2)) \\
& \leq \lambda U_{j}\left(t, b^{+}\right) & \text {(by the de-activation rule) } \\
& =U_{j}(t, b) & \text { (by property }(17))
\end{array}
$$

So agent $j$ is not deactivated in round $t$ under bid vector $b$, i.e. $j \in S_{\text {act }}(t+1, b)$, completing the proof.

(C4). Use induction on $t$. The base case $t=0$ holds because initially all agents are active. For the induction step, assume that the statement holds for some round $t \geq 0$. Suppose $i \in S_{\text {act }}(t+1, b)$. We need to prove that $i \in S_{\text {act }}\left(t+1, b^{+}\right)$.

Note that $i \in S_{\text {act }}(t, b)$, and so $i \in S_{\text {act }}\left(t, b^{+}\right)$by the induction hypothesis. Therefore

$$
\begin{aligned}
L^{*}(t, b) & \leq U_{i}(t, b) & \text { (by the de-activation rule) } \\
& =\lambda U_{i}\left(t, b^{+}\right) & \text {(by property (17)). }
\end{aligned}
$$


If $L^{*}(t, b)=\lambda L^{*}\left(t, b^{+}\right)$, then $L^{*}\left(t, b^{+}\right)=L^{*}(t, b) / \lambda \leq U_{i}\left(t, b^{+}\right)$, and we are done.

From here on, assume $L^{*}(t, b) \neq \lambda L^{*}\left(t, b^{+}\right)$. Then $L^{*}(t, b)<\lambda L^{*}\left(t, b^{+}\right)$by Part (C2). Pick agent $j$ which maximizes $L_{j}\left(t, b^{+}\right)$. If $j \neq i$ then $j \in S_{\text {act }}(t, b)$ by Part (C3), so

$$
\begin{aligned}
L^{*}(t, b) & \geq L_{j}(t, b) & \\
& =\lambda L_{j}\left(t, b^{+}\right) & \text {by property (17) }) \\
& =\lambda L^{*}\left(t, b^{+}\right) & \text {(by the choice of } j),
\end{aligned}
$$

contradicting our assumption. Then $j=i$, and so $L^{*}\left(t, b^{+}\right)=L_{i}\left(t, b^{+}\right) \leq U_{i}\left(t, b^{+}\right)$.

Ex-post monotonicity follows easily from (C3-C4).

Claim 7.13. Consider a fixed round $t$. Suppose agent $i$ is allocated with bid vector $b$. Then it is also allocated with bid vector $b^{+}$.

Proof. Since $i \in S_{\text {act }}(t, b)$, by (C4) we have $i \in S_{\text {act }}\left(t, b^{+}\right)$.

If agent $i$ is the designated agent in round $t$, then as such it is allocated under both bid vectors. If agent $i$ is not the designated agent in round $t$, then $i=\min S_{\text {act }}(t, b)$. By (C3) it holds that $S_{\text {act }}\left(t, b^{+}\right) \subset S_{\text {act }}(t, b)$, which implies that $i=\min S_{\text {act }}\left(t, b^{+}\right)$. So $i$ is allocated under bid vector $b^{+}$, too.

Regret analysis. The regret analysis is relatively standard, following the ideas in [Auer et al. 2002a]. For simplicity assume that $b_{\max }=1$. Fix a bid vector $b$.

For each agent $i$, let $c_{i}(t)$ and $n_{i}(t)$ be, respectively, the number of clicks and impressions in all rounds $s \leq t$ when it is allocated as the designated agent. Let $r_{i}(t)=\sqrt{8 \log (T) / n_{i}(t)}$. Then the event

$$
\left|\mu_{i}-c_{i}(t) / n_{i}(t)\right| \leq r_{i}(t) \text { for all rounds } t
$$

holds with probability at least $1-T^{-2}$.16 In what follows, let us assume that this event holds for all agents $i$. (The regret accumulated if this event fails is negligible.)

Then it easily follows from the specs of NewCB that for each agent $i$,

$$
\left\{\begin{array}{l}
L_{i}(t, b) \leq b_{i} \mu_{i} \leq U_{i}(t, b) \\
U_{i}(t, b)-L_{i}(t, b) \leq 2 r_{i}(t)
\end{array}\right.
$$

Let $i^{*} \in \operatorname{argmax}_{i} b_{i} \mu_{i}$ be a best agent. Note that $U_{i^{*}}(t, b) \geq b_{i^{*}} \mu_{i^{*}} \geq b_{i} \mu_{i} \geq L_{i}(b, t)$ for all agents $i$ and rounds $t$. It follows that $i^{*}$ is never de-activated by the algorithm.

Consider some agent $i$ with $\Delta_{i} \triangleq b_{i^{*}} \mu_{i^{*}}-b_{i} \mu_{i}>0$. Then $r_{i}(t)<\Delta_{i}$ after $O\left(\Delta_{i}^{-2} \log T\right)$ rounds in which this agent is allocated as the designated agent. After such round $t$,

$$
U_{i}(b, t) \leq b_{i} \mu_{i}+r_{i}(t)<b_{i} \mu_{i}+\Delta_{i}=b_{i^{*}} \mu_{i^{*}} \leq L_{i *}(b, t),
$$

and therefore agent $i$ is deactivated. It follows that agent $i$ is allocated as the designated agent at most $O\left(\Delta_{i}^{-2} \log T\right)$ times. Therefore it is de-activated after at most $O\left(k \Delta_{i}^{-2} \log T\right)$ rounds. This, in turn, implies the claimed regret bound.

\footnotetext{
${ }^{16}$ This follows from Azuma-Hoeffding inequality via a standard argument, one version of which we provide below. Fix agent $i$. For each $s \in N$, let $X_{s}$ be the click bit for the $s$-th time this agent is allocated as the designated agent, if $s \leq n_{i}(T)$, and otherwise define $X_{s}$ to be an independent $0-1$ random variable with expectation $\mu_{i}$. Then the random variables $Y_{s}=X_{s}-\mu_{s}, s \in \mathbb{N}$ form a martingale. Applying AzumaHoeffding inequality to $Y_{1}, \ldots, Y_{N}$, for any given $N$, we obtain that the event $\left|\sum_{s=1}^{N} Y_{s}\right| \leq \sqrt{8 N \log (T)}$ holds with probability at least $1-T^{-3}$. Taking the Union Bounds over all $N \leq T$, and noting that $c_{i}(t)=$ $\sum_{s=1}^{n_{i}(t)} X_{s}$, it follows that the event $\left[20\right.$ holds with probability at least $1-T^{-2}$.
} 


\subsection{The power of randomization}

A by-product of Theorem 7.9 is a separation between the power of deterministic and randomized mechanisms, in terms of regret for MAB mechanisms that are ex-post truthful and ex-post normalized. The lower bound for deterministic mechanisms is from [Babaioff et al. 2014].

One challenge here is to ensure that the upper and lower bounds talk about exactly the same problem; as stated, Theorem 7.9 and the main lower bound result from [Babaioff et al. 2014] do not. To bypass this problem, we focus on the case of two agents, and use a more general version of the lower bound: Theorem C.1 in the full version of [Babaioff et al. 2014]. Further, to match [Babaioff et al. 2014] we extend the mechanism from Theorem 7.9 to a setting in which $b_{\max }$ is not known a priori.

We formulate the separation theorem as follows. Denote $R\left(T, b_{\max }\right) \triangleq \max R(T ; b ; \mu)$, where the maximum is taken over all CTR vectors $\mu$ and all bid vectors $b$ such that $b_{i} \leq b_{\max }$ for all $i$.

THEOREM 7.14. Consider the stochastic MAB mechanism design problem with two agents. Assume $b_{\max }$ is not known a priori to the mechanism. Suppose $\mathcal{M}$ is an $M A B$ mechanism that is (i) ex-post truthful and ex-post normalized, and (ii) has regret $R\left(T, b_{\max }\right)=\tilde{O}\left(b_{\max } T^{\gamma}\right)$ for some $\gamma$ and any $b_{\max }$. Then:

(a) Babaioffet al. 2014] If $\mathcal{M}$ is deterministic then $\gamma \geq \frac{2}{3}$.

(b) There exists such randomized $\mathcal{M}$ with $\gamma=\frac{1}{2}$.

PROOF OF PART (B). Let $\mathcal{A}^{\prime}$ be the ex-post monotone MAB allocation rule in Theorem 7.9, for $b_{\max }=1$. Define an $\mathrm{MAB}$ allocation rule $\mathcal{A}$ as a rule that inputs the bid vector $b$ and passes the modified bid vector $b^{\prime}=b /\left(\max _{i} b_{i}\right)$ to $\mathcal{A}^{\prime}$. We claim that $\mathcal{A}$ is expost monotone, too. Indeed, w.l.o.g. assume $b_{1}>b_{2}$. If $b_{2}$ increases (to a value $\leq b_{1}$ ), then $b_{2}^{\prime}$ increases while $b_{1}^{\prime}$ stays the same. Thus, the total click reward of agent 2 increases. If $b_{1}$ increases then $b_{2}^{\prime}$ decreases while $b_{1}^{\prime}$ stays the same, so the total click reward of agent 2 does not increase, which implies that the total click reward of agent 1 does not decrease. Claim proved. Now part (b) follows from Theorem 7.1 (with $\mu=\frac{1}{T}$ ).

\section{EXTENSION TO MULTI-PARAMETER DOMAINS}

Our general transformation from Section 4 can be extended to multi-parameter mechanisms.It is known that a multi-parameter allocation rule is truthfully implementable if and only if it satisfies a property called "cycle-monotonicity". Similar to the singleparameter case, we present a general procedure to take any cycle-monotone allocation rule $\mathcal{A}$ and transform it into a randomized mechanism that is truthful-in-expectation, implements the same outcome as $\mathcal{A}$ with probability arbitrarily close to 1 , and requires evaluating that allocation rule only once. The technical contribution here is that we find a reduction from the multi-parameter setting to the single-parameter case.

This section is self-contained. For more background on multi-parameter mechanisms for a CS-oriented audience, please refer to [Archer and Kleinberg 2008a,b]. An Economics-oriented background for this area can be found in [Ashlagi et al. 2010].

\subsection{Preliminaries: multi-parameter domains}

Generalized types. In the full generality, multi-parameter mechanisms are defined as follows. There are $n$ agents and a set $\mathcal{O}$ of outcomes. Each agent $i$ is characterized by his type $\mathbf{x}_{i}: \mathcal{O} \rightarrow \mathbb{R}$, where $\mathbf{x}_{i}(o)$ is interpreted as the agent's valuation for the outcome $o \in \mathcal{O}$. For each agent $i$ there is a set of feasible types, denoted $\mathcal{T}_{i}$. Denote 
$\mathcal{T}=\mathcal{T}_{1} \times \ldots \times \mathcal{T}_{n}$ and call it the type space; call $\mathcal{T}_{i}$ the type space of agent $i$. The mechanism knows $(n, \mathcal{O}, \mathcal{T})$, but not the actual types $\mathbf{x}_{i}$; each type $\mathbf{x}_{i}$ is known only to the corresponding agent $i$. Formally, a problem instance, also called a multi-parameter domain, is a tuple $(n, \mathcal{O}, \mathcal{T})$.

Using this general notion of types, we define truthful mechanisms in essentially the same way as in Section 3 , with minimal syntactic changes. A (direct revelation) mechanism $\mathcal{M}$ consists of the pair $(\mathcal{A}, \mathcal{P})$, where $\mathcal{A}: \mathcal{T} \rightarrow \mathcal{O}$ is the allocation rule and $\mathcal{P}: \mathcal{T} \rightarrow \mathbb{R}^{n}$ is the payment rule. Both $\mathcal{A}$ and $\mathcal{P}$ can be randomized. Each agent $i$ reports a type $\mathbf{b}_{i} \in \mathcal{T}_{i}$ to the mechanism, which is called the bid of this agent. We denote the vector of bids by $\mathbf{b}=\left(\mathbf{b}_{1}, \ldots, \mathbf{b}_{n}\right) \in \mathcal{T}$. The mechanism receives the bid vector $\mathbf{b} \in \mathcal{T}$, selects an outcome $\mathcal{A}(\mathbf{b})$, and charges each agent $i$ a payment of $\mathcal{P}_{i}(\mathbf{b})$. The utilities are quasi-linear and agents are risk-neutral: if agent $i$ has type $\mathbf{x}_{i} \in \mathcal{T}_{i}$ and the bid vector is $\mathbf{b} \in \mathcal{T}$, then this agent's utility is

$$
u_{i}\left(\mathbf{x}_{i} ; \mathbf{b}\right)=\underset{\mathcal{M}}{\mathbb{E}}\left[\mathbf{x}_{i}(\mathcal{A}(\mathbf{b}))-\mathcal{P}_{i}(\mathbf{b})\right]
$$

For each type $\mathbf{x}_{i} \in \mathcal{T}_{i}$ of agent $i$ we use a standard notation $\left(\mathbf{b}_{-i}, \mathbf{x}_{i}\right)$ to denote the bid vector $\hat{\mathbf{b}}$ such that $\hat{\mathbf{b}}_{i}=\mathbf{x}_{i}$ and $\hat{\mathbf{b}}_{j}=\mathbf{b}_{j}$ for every agent $j \neq i$.

Special case: dot-product valuations. For intuition, consider dot-product valuations, an important special case where the type $\mathrm{x} \in \mathcal{T}_{i}$ of each agent $i$ can be decomposed as a dot product $\mathbf{x}(o)=\beta_{\mathbf{x}} \cdot a_{i}(o)$, for each outcome $o \in \mathcal{O}$, where $\beta_{\mathbf{x}}, a_{i}(o) \in \mathbb{R}^{d}$ are some finite-dimensional vectors. Here the term $a_{i}(o)$ is the same for all types $\mathbf{x} \in \mathcal{T}_{i}$ (and known to the mechanism), whereas $\beta_{\mathbf{x}}$ is the same for all outcomes $o \in \mathcal{O}$ and is known only to agent $i$. The term $a_{i}(o)$ is usually called an "allocation" of agent $i$ for outcome $o$, and $\beta_{\mathbf{x}}$ is called the "private value". The single-parameter domains defined in Section 3 correspond to the case $d=1$.

Note that the type $\mathrm{x}$ of each agent $i$ is determined by the corresponding private value $\beta_{\mathbf{x}}$, and his type space $\mathcal{T}_{i}$ is determined by $D_{i}=\left\{\beta_{\mathbf{x}}: \mathbf{x} \in \mathcal{T}_{i}\right\} \subset \mathbb{R}^{d}$. Because of this, in the literature on dot-product valuations the term "type" often refers to $\beta_{\mathbf{x}}$. To avoid ambiguity, in this section we will refer to $\beta_{\mathbf{x}}$ as "private value" rather than "type", and call $D_{1} \times \ldots \times D_{n}$ the private value space.

Game-theoretic properties. Truthfulness and individual rationality are defined exactly as in Section 3 if expressed in terms of the agents' utility:

-A mechanism is truthful if for every agent $i$ truthful bidding is a dominant strategy:

$$
u_{i}\left(\mathbf{x}_{i} ;\left(\mathbf{b}_{-i}, \mathbf{x}_{i}\right)\right) \geq u_{i}\left(\mathbf{x}_{i} ; \mathbf{b}\right) \quad \forall \mathbf{x}_{i} \in \mathcal{T}_{i}, \mathbf{b} \in \mathcal{T} .
$$

An allocation rule is called truthfully implementable if it is the allocation rule in some truthful mechanism.

- A mechanism is individually rational (IR) if each agent $i$ never receives negative utility by participating in the mechanism and bidding truthfully:

$$
u_{i}\left(\mathbf{x}_{i} ;\left(\mathbf{b}_{-i}, \mathbf{x}_{i}\right)\right) \geq 0 \quad \forall \mathbf{x}_{i} \in \mathcal{T}_{i}, \mathbf{b}_{-i} \in \mathcal{T}_{-i} .
$$

The right-hand side in (23) represents the maximal guaranteed utility of an "outside option" (i.e., from not participating in the mechanism). For example, our definition of IR is meaningful whenever this utility is 0 , which is a typical assumption for most multi-parameter domains studied in the literature.

Our assumptions. We make two assumptions on the type space $\mathcal{T}$ :

- non-negative types: $\mathbf{x}_{i}(o) \geq 0$ for each agent $i$, each type $\mathbf{x}_{i} \in \mathcal{T}_{i}$, and each outcome $o \in \mathcal{O}$. 
- rescalable types: $\lambda \mathbf{x}_{i} \in \mathcal{T}_{i}$ for each agent $i$, each type $\mathbf{x}_{i} \in \mathcal{T}_{i}$, and any parameter $\lambda \in[0,1]$.

For dot-product valuations, types are rescalable if and only if it holds that $\beta_{\mathbf{x}} \in D_{i} \Rightarrow$ $\lambda \beta_{\mathbf{x}} \in D_{i}$ for each $\lambda \in[0,1]$. Thus, assuming rescalable types is equivalent to assuming that the set $D_{i}$ is star-convex at 0 . To ensure non-negative types, it suffices to assume that $D_{i} \subset \mathbb{R}_{+}^{d}$ for each agent $i$, and all allocations are non-negative: $a_{i}(o) \in \mathbb{R}_{+}^{d}$ for all $o \in \mathcal{O}$.

In particular, for each agent $i$ there exists a zero type: a type $\mathbf{x}_{i} \in \mathcal{T}_{i}$ such that $\mathbf{x}_{i}(\cdot) \equiv 0$. Let us say that a mechanism is normalized if for each agent $i$, the expected payment of this agent is 0 whenever she submits the zero type.

Truthfulness characterization. We will use the following characterization of truthful mechanisms. A (randomized) allocation rule $\mathcal{A}$ is cycle-monotone if the following property holds: for each bid vector $\mathbf{b} \in \mathcal{T}$, each agent $i$, each $k \geq 2$, and each $k$-tuple $\mathbf{x}_{i, 0}, \mathbf{x}_{i, 1}, \ldots, \mathbf{x}_{i, k} \in \mathcal{T}_{i}$ of this agent's types, we have

$$
\underset{\mathcal{A}}{\mathbb{E}}\left[\sum_{j=0}^{k} \mathbf{x}_{i, j}\left(o_{i, j}\right)-\mathbf{x}_{i,(j-1) \bmod k}\left(o_{i, j}\right)\right] \geq 0, \quad \text { where } o_{i, j}=\mathcal{A}\left(\mathbf{b}_{-i}, \mathbf{x}_{i, j}\right) \in \mathcal{O}
$$

THEOREM 8.1 (ROCHET [1987]). Consider an arbitrary multi-parameter domain $(n, \mathcal{O}, \mathcal{T}) . A$ (randomized) allocation rule $\mathcal{A}$ is truthfully implementable if and only if it is cycle-monotone. Assuming rescalable types, for any cycle-monotone allocation rule $\mathcal{A}$, a mechanism $(\mathcal{A}, \mathcal{P})$ is truthful and normalized if and only if

$$
\underset{\mathcal{A}}{\mathbb{E}}\left[\mathcal{P}_{i}(\mathbf{b})\right]=\underset{\mathcal{A}}{\mathbb{E}}\left[\mathbf{b}_{i}(\mathcal{A}(\mathbf{b}))-\int_{t=0}^{1} \mathbf{b}_{i}\left(\mathcal{A}\left(\mathbf{b}_{-i}, t \mathbf{b}_{i}\right)\right) d t\right] .
$$

Note that this theorem generalizes Theorem 3.2 for single-parameter mechanisms, as applied to single-parameter domains with private value space $[0,1]^{n}$. In particular, Equation (25) generalizes the Myerson payment rule for single-parameter mechanisms.

\subsection{The multi-parameter transformation}

Consider allocation rule $\mathcal{A}$, bid vector $\mathbf{b} \in \mathcal{T}$, and the rescaling vector $\lambda \in[0,1]^{n}$. Denote

$$
\lambda \otimes b=\left(\lambda_{1} \mathbf{b}_{1}, \ldots, \lambda_{n} \mathbf{b}_{n}\right) \in \mathcal{T} .
$$

In other words, $\lambda \otimes b$ is the "rescaled" bid vector where the bid of each agent $i$ is $\lambda_{i} \mathbf{b}_{i}$; this bid vector is well-defined because we assumed the rescalable types property. Note that for each $b$ the subset

$$
\mathcal{T}_{\mathbf{b}}=\left\{\lambda \otimes b: \lambda \in[0,1]^{n}\right\} \subset \mathcal{T}
$$

forms a single-parameter type space where each agent $i$ has private value $\lambda_{i} \in[0,1]$ and allocation $b_{i}(o)$ for every outcome $o$. By abuse of notation, let us treat the allocation / payment rules for $\mathcal{T}_{\mathbf{b}}$ as functions from the private value space $[0,1]^{n}$ rather than the type space $\mathcal{T}_{\mathbf{b}}$.

Consider an allocation rule $\mathcal{A}_{\mathbf{b}}(\lambda)=\mathcal{A}(\lambda \otimes \mathbf{b})$ for the single-parameter type space $\mathcal{T}_{\mathrm{b}}$. If the original allocation rule $\mathcal{A}$ is truthfully implementable for type space $\mathcal{T}$ using payment rule $\mathcal{P}$, then $\mathcal{A}_{\mathbf{b}}$ is truthfully implementable for type space $\mathcal{T}_{\mathbf{b}}$ using payment rule $\mathcal{P}_{\mathbf{b}}(\lambda)=\mathcal{P}(\lambda \otimes \mathbf{b})$, because restricting the allocation and payment rules to $\mathcal{T}_{\mathbf{b}}$ only limits the set of possible misreports of an agent. Essentially, the idea will be to apply our single-parameter transformation to $\mathcal{A}_{\mathrm{b}}$. 
Let $\mathbf{f}_{\mu}=\left(f_{1}, \ldots, f_{n}\right)$ be the $n$-tuple of canonical self-resampling procedures with resampling probability $\mu$, for some fixed $\mu \in(0,1)$. (See Algorithm 1 on page 13,) For each bid vector $\mathbf{b} \in \mathcal{T}$, let

$$
\left(\widetilde{\mathcal{A}}_{\mathbf{b}}, \widetilde{\mathcal{P}}_{\mathbf{b}}\right)=\operatorname{AllocToMech}\left(\mathcal{A}_{\mathbf{b}}, \mu, \mathbf{f}_{\mu}\right)
$$

be the single-parameter mechanism for type space $\mathcal{T}_{\mathrm{b}}$ obtained by applying our singleparameter transformation from Section 4 to allocation $\mathcal{A}_{\mathrm{b}}[17$

The transformed multi-parameter mechanism is defined as

$$
(\widetilde{\mathcal{A}}(\mathbf{b}), \widetilde{\mathcal{P}}(\mathbf{b}))=\left(\widetilde{\mathcal{A}}_{\mathbf{b}}(\overrightarrow{1}), \widetilde{\mathcal{P}}_{\mathbf{b}}(\overrightarrow{1})\right) \text { for every } b \in \mathcal{T} \text {. }
$$

This completes the description of our multi-parameter transformation. The useful properties of this transformation are captured in the theorem below.

THEOREM 8.2. Consider an arbitrary multi-parameter domain $(n, \mathcal{O}, \mathcal{T})$ with rescalable, non-negative types. Let $\mathcal{A}$ be a cycle-monotone allocation rule. Let $\mathcal{M}_{\mu}=$ $(\widetilde{\mathcal{A}}, \widetilde{\mathcal{P}})$ be the transformed mechanism defined by Equations (26] [27), for some parameter $\mu \in(0,1)$. Then $\mathcal{M}_{\mu}$ has the following properties:

(a) $\mathcal{M}_{\mu}$ is truthful and normalized.

(b) $\mathcal{M}_{\mu}$ is universally ex-post individually rational and ex-post no-positive-transfers. Moreover, given a bid vector $\mathbf{b}$, it never pays any agent $i$ more than $\mathbf{b}_{i}(o)\left(\frac{1}{\mu}-1\right)$, where $o=\mathcal{A}(\mathbf{b}) \in \mathcal{O}$.

(c) For any bid vector $\mathbf{b} \in \mathcal{T}$ (and any fixed random seed of nature) allocations $\widetilde{\mathcal{A}}(\mathbf{b})$ and $\mathcal{A}(\mathbf{b})$ are identical with probability at least $1-n \mu$.

(d) If $\mathcal{A}$ is $\alpha$-approximate (for social welfare) then $\widetilde{\mathcal{A}}$ is $\alpha /\left(1-\frac{2}{1-\mu}\right)$-approximate.

Proof. Parts (b) and (c) follow immediately from Theorem 4.5, and part (d) follows immediately from Theorem 5.1. Thus, it remains to prove part (a).

Note that the single-parameter allocation rule $\widetilde{\mathcal{A}}_{\mathrm{b}}$ has the following property: for each agent $i$ the single-parameter bid $\lambda_{i}$ is rescaled by the (randomly chosen) factor $\chi_{i} \in[0,1]$ which does not depend on the bid, and then $\mathcal{A}_{\mathbf{b}}$ is called. Therefore, letting $\chi=\left(\chi_{1}, \ldots, \chi_{n}\right)$, it holds that

$$
\widetilde{\mathcal{A}}_{\mathbf{b}}(\lambda)=\mathcal{A}(\chi \otimes(\lambda \otimes \mathbf{b})) \quad \text { for all } \mathbf{b} \in \mathcal{T} \text { and } \lambda \in[0,1]^{n} .
$$

We claim that $\widetilde{\mathcal{A}}$ is cycle-monotone. Indeed, fix bid vector $\mathbf{b} \in \mathcal{T}$, agent $i$, some $k \geq 2$, and a $k$-tuple $\mathbf{x}_{i, 0}, \mathbf{x}_{i, 1}, \ldots, \mathbf{x}_{i, k} \in \mathcal{T}_{i}$ of this agent's types. Let us consider a fixed realization of the random vector $\chi \in[0,1]^{n}$. For each type $\mathbf{x}_{i, j}$, note that (by Equations (28) and (27)) we have

$$
\widetilde{\mathcal{A}}\left(\mathbf{x}_{i, j}, \mathbf{b}_{-i}\right)=\widetilde{\mathcal{A}}_{\left(\mathbf{x}_{i, j}, \mathbf{b}_{-i}\right)}(\overrightarrow{1})=\mathcal{A}\left(\chi \otimes\left(\mathbf{b}_{-i}, \mathbf{x}_{i, j}\right)\right) \in \mathcal{O} .
$$

Denote this outcome by $o_{i, j}(\chi)$. Apply the cycle-monotonicity of $\mathcal{A}$ for bid vector $\chi \otimes$ $\left(\mathbf{x}_{i, j}, \mathbf{b}_{-i}\right)$ :

$$
\underset{\mathcal{A}}{\mathbb{E}}\left[\sum_{j=0}^{k} \mathbf{x}_{i, j}\left(o_{i, j}(\chi)\right)-\mathbf{x}_{i,(j-1) \bmod k}\left(o_{i, j}(\chi)\right)\right] \geq 0
$$

\footnotetext{
${ }^{17}$ Note that the transformed mechanism depends on $\mu$. We do not make this dependence explicit, to simplify the notation.
} 
Recalling that $o_{i, j}(\chi)=\widetilde{\mathcal{A}}\left(\mathbf{x}_{i, j}, \mathbf{b}_{-i}\right)$, we observe that for this fixed realization of $\chi$, Equation (29) is exactly the inequality in the definition of cycle-monotonicity for $\widetilde{\mathcal{A}}$. Therefore taking expectation over $\chi$, we obtain the desired inequality (24) for $\widetilde{\mathcal{A}}$. Claim proved 18

It remains to prove that in the transformed mechanism $(\widetilde{\mathcal{A}}, \widetilde{\mathcal{P}})$, the payment rule satisfies Equation (25). Fix bid vector $\mathbf{b}$ and consider the transformed single-parameter mechanism $\left(\widetilde{\mathcal{A}}_{\mathbf{b}}, \widetilde{\mathcal{P}}_{\mathbf{b}}\right)$ for the single-parameter type space $\mathcal{T}_{\mathbf{b}}$. In the terminology of single-parameter domains, each agent $i$ receives an allocation $\widetilde{\mathcal{A}}_{\mathbf{b}, i}(\lambda)=b_{i}\left(\widetilde{\mathcal{A}}_{\mathbf{b}}(\lambda)\right)$ whenever the bid vector is $\lambda \in[0,1]^{n}$. Since this is a truthful and normalized singleparameter mechanism, it follows that

$$
\mathbb{E}\left[\widetilde{\mathcal{P}}_{\mathbf{b}}(\lambda)\right]=\mathbb{E}\left[\lambda_{i} \widetilde{\mathcal{A}}_{\mathbf{b}, i}(\lambda)-\int_{0}^{\lambda_{i}} \widetilde{\mathcal{A}}_{\mathbf{b}, i}\left(\lambda_{-i}, u\right) d u\right], \quad \forall \lambda \in[0,1]^{n} .
$$

Plugging in $\lambda=\overrightarrow{1}$ and Equation (27), we obtain the desired Equation (25).

\section{OPEN QUESTIONS}

This paper gives rise to a number of open questions. As discussed in Section 2.1, some of these questions have been partially addressed in the follow-up work. Here we present the current status.

Variance vs. expectation tradeoff. Randomized mechanisms constructed via our general transformation exhibit an explicit tradeoff between the variance in payments and the loss in expected welfare compared to the optimal allocation rule. Since the variance in payments can be very high, it is desirable to optimize this tradeoff (to complement the expectation-only guarantees).

The worst-case optimality result in Wilkens and Sivan [2012], discussed in Section 2.1, does not resolve this question, since it does not rule out a reduction which achieves a better tradeoff for some (but not all) monotone allocation rules. Further, the optimal tradeoff for a given domain could be achieved by a mechanism that cannot be presented as a reduction from some welfare-optimal allocation rule.

Our informal conjecture is that the tradeoff in this paper is optimal for any given single-parameter domain with "informational obstacle", i.e. whenever payment computation for welfare-optimal allocation rule is impossible due to the insufficient observable information.

A specific formal conjecture is that our tradeoff is optimal for MAB mechanisms. To take an extreme version, what welfare loss can be achieved if no rebates (i.e., no positive transfers) are allowed?

The power of randomization. We have a separation result for randomized vs. deterministic ex-post truthful MAB mechanisms. Can one obtain similar separation results for other single-parameter domains? The positive side for any such hypothetical separation result is provided by our general reduction, so it remains to produce the corresponding negative result for deterministic mechanisms. However, such negative results are not likely to be easy, considering the difficulties faced by [Babaioff et al. 2014; Devanur and Kakade 2009] for MAB mechanisms. One specific target would be the router scheduling problem proposed in [Shnayder et al. [2012].

\footnotetext{
${ }^{18}$ Note that the proof of cycle-monotonicity of $\widetilde{\mathcal{A}}$ did not use any other property of the canonical selfresampling procedures $\mathbf{f}_{\mu}$ other than Equation [28. The truthfulness properties of $\mathbf{f}_{\mu}$ are used in the forthcoming argument about payments.
} 
MAB allocation rules. This paper opens up the problem of designing monotone MAB allocation rules, which is a new angle in the rich literature on MAB (also see [Slivkins 2011b]). While we have focused on stochastic MAB, many other MAB settings have been studied in the literature, making various assumptions on payoff evolution over time (e.g., [Auer et al. 2002b; Slivkins and Upfal|2008; Hazan and Kale 2009]), dependencies between arms (e.g., [Flaxman et al. 2005; Pandey et al. 2007; Kleinberg et al. 2008b; [Srinivas et al. 2010]), and side information available to the algorithm (e.g., [Kleinberg et al. 2008b; Langford and Zhang 2007; Slivkins 2011a]). For most such settings one could meaningfully define the corresponding mechanism design problem; we have reduced this problem to that of designing monotone MAB allocation rules. In particular, for any given $\mathrm{MAB}$ setting one could ask whether monotone $\mathrm{MAB}$ allocation rules can achieve optimal regret.

One appealing target here is the adversarial MAB setting (with oblivious adversary). The ex-post truthful mechanism in [Babaioff et al. 2014] achieves regret $\tilde{O}\left(k^{1 / 3} T^{2 / 3}\right)$ for this setting, whereas the best known MAB algorithms achieve regret $O(\sqrt{k T})$ Auer et al. 2002b; Audibert and Bubeck 2010]; it is not clear what is the tight regret bound.

More applications. In addition to the applications presented in this paper and the follow-up work, what other domains can our general reduction (and the multiparameter extension thereof) be fruitfully applied to? In particular, one could consider two generalizations of MAB mechanisms: to multiple ads per agent and to multiple ad slots with slot-dependent values-per-click.

\section{APPENDIX: ONESHOT is equivalent to Algorithm 1 (proof of Proposition 4.8)}

Let us compare the sampling procedures defined by Algorithm 1 and ONESHOT. To simplify the notation, we will omit the subscript $i$ from the description of the procedures. That is, a self-resampling procedure inputs a scalar bid $b$ and a random seed $w$, and outputs two numbers $(x, y)$. To prove that ONESHOT is equivalent to Algorithm 1 , we analyze a family of sampling rules that uses bounded-depth recursion to "interpolate" between ONESHOT and Algorithm 1. Specifically, define $\mathrm{BDR}_{k}$ to be the following family of sampling algorithms parameterized by $k \in \mathbb{N} \cup\{\infty\}$, where $k-1$ is interpreted as $\infty$ when $k=\infty$.

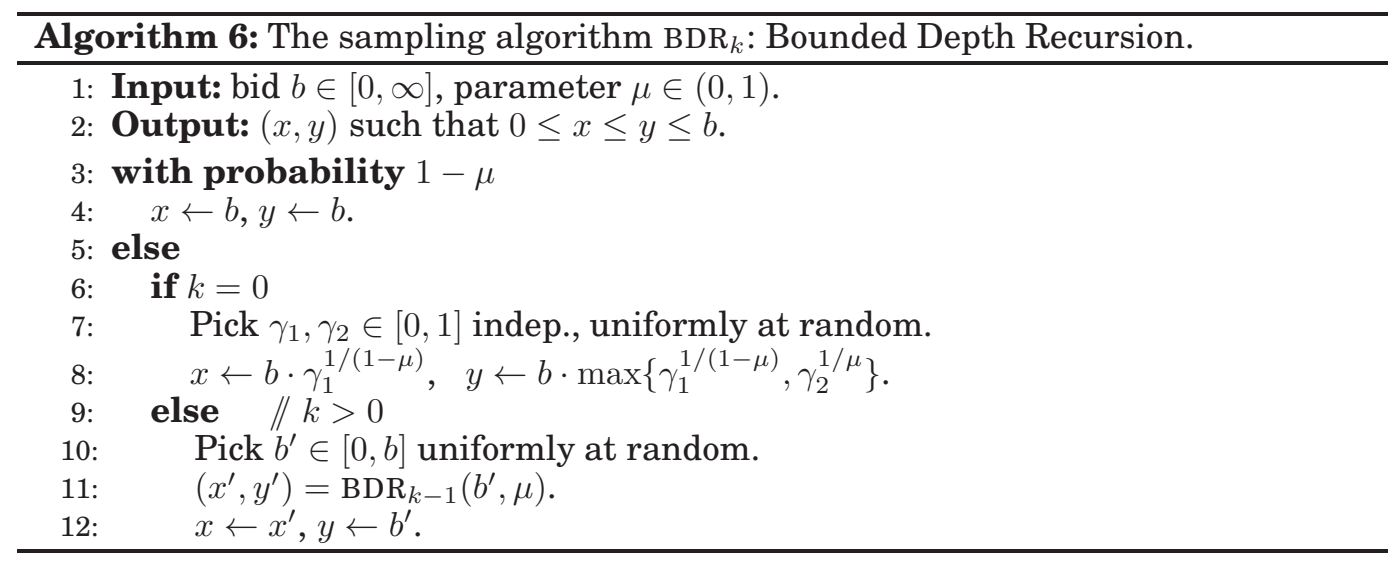


The reader may easily verify that $\mathrm{BDR}_{k}$ is equal to ONESHOT when $k=0$ and that it is equal to Algorithm 1 when $k=\infty$. Furthermore, for any $k<k^{\prime}$ (where $k^{\prime} \leq \infty$ ) there is an obvious coupling of $\mathrm{BDR}_{k}$ with $\mathrm{BDR}_{k^{\prime}}$ such that the two algorithms have probability at most $\mu^{k+1}$ of outputting different results: simply let the two executions share the same randomness until the recursion depth equals $k$. Thus, as $k \rightarrow \infty$, the output distribution of $\mathrm{BDR}_{k}$ converges, in total variation distance, to that of $\mathrm{BDR}_{\infty}$. We will prove that for every finite $k$ the algorithms $\mathrm{BDR}_{k}$ and $\mathrm{BDR}_{k+1}$ have identical output distributions, from which it follows that their output distribution is identical to that of $\mathrm{BDR}_{0}$ and, therefore, that $\mathrm{BDR}_{\infty}$ also has the same output distribution as $\mathrm{BDR}_{0}$, confirming Proposition 4.8 .

Couple $\mathrm{BDR}_{k}$ and $\mathrm{BDR}_{k+1}$ so that they use shared randomness until the two algorithms reach differing points in their control flow. This occurs when the first algorithm is executing a call to $\mathrm{BDR}_{0}$ and the second algorithm is executing a call to $\mathrm{BDR}_{1}$ on the same input $(\beta, \mu)$. (We are denoting the input in this step of the recursive algorithms by $(\beta, \mu)$ rather than $(b, \mu)$, to distinguish $\beta$ from the value of $b$ on which the two algorithms $\mathrm{BDR}_{k}, \mathrm{BDR}_{k+1}$ were originally called.) At this point, with probability $1-\mu$ both algorithms output $(x, y)=(\beta, \beta)$. Conditional on this event not taking place, $\operatorname{BDR}_{0}$ outputs $(x, y)=\left(\gamma_{1}^{1 / p} \beta, \max \left\{\gamma_{1}^{1 / p}, \gamma_{2}^{1 / q}\right\} \beta\right)$ where $p=1-\mu, q=\mu$. Instead $\mathrm{BDR}_{1}$ computes $\beta^{\prime}=\gamma_{3} \beta$ where $\gamma_{3} \in[0,1]$ is uniformly random, and it outputs $(x, y)=\left(\beta^{\prime}, \beta^{\prime}\right)$ with probability $p$ and otherwise $(x, y)=\left(\gamma_{1}^{1 / p} \beta^{\prime}, \beta^{\prime}\right)$. Lemma A.1 tells us that these two output distributions are the same.

LEMMA A.1. Let $\gamma_{1}, \gamma_{2}, \gamma_{3}$ be mutually independent random variables, each uniformly distributed in $[0,1]$. Let $p, q>0$ be numbers such that $p+q=1$. Define random variables $x, y, z$ by:

$$
\begin{aligned}
& x=\gamma_{1}^{1 / p} \\
& y=\max \left\{\gamma_{1}^{1 / p}, \gamma_{2}^{1 / q}\right\} \\
& z= \begin{cases}\gamma_{3} & \text { if } \gamma_{2}<p \\
\gamma_{1}^{1 / p} \gamma_{3} & \text { if } \gamma_{2} \geq p\end{cases}
\end{aligned}
$$

Then the pairs $(x, y)$ and $\left(z, \gamma_{3}\right)$ are identically distributed.

Proof. We will show, equivalently, that the pairs $(y, x / y)$ and $\left(\gamma_{3}, z / \gamma_{3}\right)$ are identically distributed. The distribution of $\left(\gamma_{3}, z / \gamma_{3}\right)$ is completely characterized by the following facts which are immediate from the definition of $z$.

(1) $\gamma_{3}$ and $z / \gamma_{3}$ are independent;

(2) $\gamma_{3}$ is uniformly distributed in $[0,1]$;

(3) $z / \gamma_{3}$ is equal to 1 with probability $p$, and conditional on $z / \gamma_{3} \neq 1$, the distribution of $\left(z / \gamma_{3}\right)^{p}$ is uniform on $[0,1)$.

To finish the proof of the lemma, we shall prove the corresponding facts about $y$ and $x / y$. Let $I_{1}, I_{2} \subseteq[0,1]$ be any pair of intervals (open, closed, or half-open). Let $a, b$ be the endpoints of $I_{1}$ and $c, d$ the endpoints of $I_{2}$. To compute $\operatorname{Pr}\left(y \in I_{1}, x / y \in I_{2}\right)$ it suffices 
to make the following two observations:

$$
\begin{aligned}
\operatorname{Pr}\left(y \in I_{1}, x / y=1\right) & =\operatorname{Pr}\left(a \leq \gamma_{1}^{1 / p} \leq b, 0 \leq \gamma_{2}^{1 / q} \leq \gamma_{1}^{1 / p}\right) \\
& =\operatorname{Pr}\left(a^{p} \leq \gamma_{1} \leq b^{p}, 0 \leq \gamma_{2} \leq \gamma_{1}^{q / p}\right) \\
& =\int_{a^{p}}^{b^{p}} t^{q / p} d t=\int_{a^{p}}^{b^{p}} t^{1 / p-1} d t=p(b-a) \\
\operatorname{Pr}\left(y \in I_{1}, x / y \in I_{2} \backslash\{1\}\right) & =\operatorname{Pr}\left(a \leq \gamma_{2}^{1 / q} \leq b, c \gamma_{2}^{1 / q} \leq \gamma_{1}^{1 / p}<d \gamma_{2}^{1 / q}\right) \\
& =\operatorname{Pr}\left(a^{q} \leq \gamma_{2} \leq b^{q}, c^{p} \gamma_{2}^{p / q} \leq \gamma_{1} \leq d^{p} \gamma_{2}^{p / q}\right) \\
& =\int_{a^{q}}^{b^{q}}\left(d^{p}-c^{p}\right) t^{p / q} d t=\left(d^{p}-c^{p}\right) \int_{a^{q}}^{b^{q}} t^{1 / q-1} d t=q\left(d^{p}-c^{p}\right)(b-a) .
\end{aligned}
$$

Therefore,

$$
\operatorname{Pr}\left(y \in I_{1}, x / y \in I_{2}\right)=(b-a) \cdot\left\{\begin{array}{ll}
q\left(d^{p}-c^{p}\right) & \text { if } 1 \notin I_{2} \\
p+q\left(d^{p}-c^{p}\right) & \text { if } 1 \in I_{2}
\end{array} .\right.
$$

From this formula it follows that $y$ and $x / y$ are independent, $y$ is uniformly distributed, $\operatorname{Pr}(x / y=1)=p$, and the distribution of $(x / y)^{p}$ conditional on $x / y \neq 1$ is uniform on $[0,1)$.

\section{Acknowledgments}

We are indebted to Tim Roughgarden for suggesting that for positive types, an improved bound on the social welfare is possible (see Section 5). We would like to acknowledge that the preliminary form of a generalization of our "generic transformation" to negative bids, and (the preliminary form of) the applications to offline mechanism design, have been derived jointly with Jason Hartline.

\section{REFERENCES}

Aaron Archer and Robert Kleinberg. 2008a. Characterizing truthful mechanisms with convex type spaces. SIGecom Exchanges 7, 3 (2008).

Aaron Archer and Robert Kleinberg. 2008b. Truthful germs are contagious: a local to global characterization of truthfulness. In 9th ACM Conf. on Electronic Commerce (EC). 21-30.

Aaron Archer, Christos Papadimitriou, Kunal Talwar, and Éva Tardos. 2004. An approximate truthful mechanism for combinatorial auctions with single parameter agents. Internet Mathematics 1 (2004), 129-150. Extended abstract in SODA 2003.

Aaron Archer and Éva Tardos. 2001. Truthful Mechanisms for One-Parameter Agents. In IEEE Symp. on Foundations of Computer Science (FOCS). 482-491.

Itai Ashlagi, Mark Braverman, Avinatan Hassidim, and Dov Monderer. 2010. Monotonicity and Implementability. Econometrica 78, 5 (2010), 1749-1772.

Susan Athey and Ilya Segal. 2013. An Efficient Dynamic Mechanism. Econometrica 81,6 (Nov. 2013), 2463-2485. A preliminary version has been available as a working paper since 2007.

J.Y. Audibert and S. Bubeck. 2010. Regret Bounds and Minimax Policies under Partial Monitoring. J. of Machine Learning Research (JMLR) 11 (2010), 2785-2836. A preliminary version has been published in COLT 2009. 
Peter Auer, Nicolò Cesa-Bianchi, and Paul Fischer. 2002a. Finite-time Analysis of the Multiarmed Bandit Problem. Machine Learning 47, 2-3 (2002), 235-256. Preliminary version in 15th ICML, 1998.

Peter Auer, Nicolò Cesa-Bianchi, Yoav Freund, and Robert E. Schapire. 2002b. The Nonstochastic Multiarmed Bandit Problem. SIAM J. Comput. 32, 1 (2002), 48-77. Preliminary version in 36th IEEE FOCS, 1995.

Moshe Babaioff, Liad Blumrosen, and Michael Schapira. 2013. The communication burden of payment determination. Games and Economic Behavior 77, 1 (2013), 153 - 167. Preliminary version in ACM EC 2008.

Moshe Babaioff, Robert Kleinberg, and Aleksandrs Slivkins. 2010. Truthful Mechanisms with Implicit Payment Computation. In 11th ACM Conf. on Electronic Commerce (EC). 43-52.

Moshe Babaioff, Robert Kleinberg, and Aleksandrs Slivkins. 2013. Multi-parameter mechanisms with implicit payment computation. In 13th ACM Conf. on Electronic Commerce (EC). 35-52.

Moshe Babaioff, Yogeshwer Sharma, and Aleksandrs Slivkins. 2014. Characterizing Truthful Multi-armed Bandit Mechanisms. SIAM J. on Computing (SICOMP) 43, 1 (2014), 194-230. Preliminary version in 10th ACM EC, 2009.

Xiaohui Bei and Zhiyi Huang. 2011. Bayesian Incentive Compatibility via Fractional Assignments. In 22nd ACM-SIAM Symp. on Discrete Algorithms (SODA). 720-733.

Dirk Bergemann and Maher Said. 2011. Dynamic Auctions: A Survey. In Wiley Encyclopedia of Operations Research and Management Science, Vol. 2. Wiley: New York, 1511-1522.

Dirk Bergemann and Juuso Välimäki. 2010. The Dynamic Pivot Mechanism. Econometrica 78, 2 (2010), 771-789. Preliminary versions have been available since 2006, as Cowles Foundation Discussion Papers \#1584 (2006), \#1616 (2007) and \#1672(2008).

Patrick Briest, Shuchi Chawla, Robert Kleinberg, and S. Matthew Weinberg. 2014. Pricing Lotteries. Journal of Economic Theory (2014). In press, accepted manuscript.

Yang Cai, Constantinos Daskalakis, and S. Matthew Weinberg. 2012. Optimal Multidimensional Mechanism Design: Reducing Revenue to Welfare Maximization. In 53th IEEE Symp. on Foundations of Computer Science (FOCS). 130-139.

Yang Cai, Constantinos Daskalakis, and S. Matthew Weinberg. 2013a. Reducing Revenue to Welfare Maximization: Approximation Algorithms and other Generalizations. In 24nd ACM-SIAM Symp. on Discrete Algorithms (SODA). 578-595.

Yang Cai, Constantinos Daskalakis, and S. Matthew Weinberg. 2013b. Understanding Incentives: Mechanism Design Becomes Algorithm Design. In 54th IEEE Symp. on Foundations of Computer Science (FOCS). 618-627.

Shuchi Chawla, Nicole Immorlica, and Brendan Lucier. 2012. On the limits of blackbox reductions in mechanism design. In 44th ACM Symp. on Theory of Computing (STOC). 435-448.

Constantinos Daskalakis and S. Matthew Weinberg. 2014. Bayesian Truthful Mechanisms for Job Scheduling from Bi-criterion Approximation Algorithms. (2014). arXiv:1405.5940.

Nikhil Devanur and Sham M. Kakade. 2009. The Price of Truthfulness for Pay-PerClick Auctions. In 10th ACM Conf. on Electronic Commerce (EC). 99-106.

Shahar Dobzinski and Shaddin Dughmi. 2009. On the Power of Randomization in Algorithmic Mechanism Design. In 50th IEEE Symp. on Foundations of Computer Science (FOCS).

Shahar Dobzinski, Hu Fu, and Robert D. Kleinberg. 2012. Optimal auctions with correlated bidders are easy. Games and Economic Behavior (2012). Special issue on selected algorithmic game theory papers from STOC, FOCS, and SODA 2011. In 
press.

Abraham Flaxman, Adam Kalai, and H. Brendan McMahan. 2005. Online Convex Optimization in the Bandit Setting: Gradient Descent without a Gradient. In 16th ACM-SIAM Symp. on Discrete Algorithms (SODA). 385-394.

Nicola Gatti, Alessandro Lazaric, and Francesco Trovo. 2012. A Truthful Learning Mechanism for Contextual Multi-Slot Sponsored Search Auctions with Externalities. In 13th ACM Conf. on Electronic Commerce (EC).

Jason D. Hartline. 2012. Approximation in Economic Design. Draft of a forthcoming book, available at http: // jasonhartline.com/MDnA/. (2012).

Jason D. Hartline, Robert Kleinberg, and Azarakhsh Malekian. 2011. Bayesian Incentive Compatibility via Matchings. In 22nd ACM-SIAM Symp. on Discrete Algorithms (SODA). 734-747.

Jason D. Hartline and Brendan Lucier. 2010. Bayesian algorithmic mechanism design. In 42th ACM Symp. on Theory of Computing (STOC). 301-310.

Elad Hazan and Satyen Kale. 2009. Better algorithms for benign bandits. In 20th ACM-SIAM Symp. on Discrete Algorithms (SODA). 38-47.

Zhiyi Huang and Sampath Kannan. 2012. Exponential mechanism for social welfare: private, truthful, and nearly optimal. Working paper. (2012).

Navendu Jain, Ishai Menache, Joseph Naor, and Jonathan Yaniv. 2011. A Truthful Mechanism for Value-Based Scheduling in Cloud Computing. In 4th Symp. on Algorithmic Game Theory (SAGT).

Robert Kleinberg, Alexandru Niculescu-Mizil, and Yogeshwer Sharma. 2008a. Regret bounds for sleeping experts and bandits. In 21st Conf. on Learning Theory (COLT). 425-436.

Robert Kleinberg, Aleksandrs Slivkins, and Eli Upfal. 2008b. Multi-Armed Bandits in Metric Spaces. In 40th ACM Symp. on Theory of Computing (STOC). 681-690.

T.L. Lai and Herbert Robbins. 1985. Asymptotically efficient Adaptive Allocation Rules. Advances in Applied Mathematics 6 (1985), 4-22.

John Langford and Tong Zhang. 2007. The Epoch-Greedy Algorithm for Contextual Multi-armed Bandits. In 21st Advances in Neural Information Processing Systems (NIPS).

Alejandro M. Manelli and Daniel R. Vincent. 2006. Bundling as an optimal selling mechanism for a multiple-good monopolist. Journal of Economic Theory 127, 1 (2006), $1-35$.

Frank McSherry and Kunal Talwar. 2007. Mechanism Design via Differential Privacy. In 48th IEEE Symp. on Foundations of Computer Science (FOCS). 94-103.

Roger B. Myerson. 1981. Optimal Auction Design. Mathematics of Operations Research 6 (1981), 58-73.

N. Nisan and A. Ronen. 2001. Algorithmic Mechanism Design. Games and Economic Behavior 35, 1-2 (2001), 166-196.

Sandeep Pandey, Deepayan Chakrabarti, and Deepak Agarwal. 2007. Multi-armed Bandit Problems with Dependent Arms. In 24th Intl. Conf. on Machine Learning (ICML).

Jean-Charles Rochet. 1987. A necessary and sufficient condition for rationalizability in a quasi-linear context. J. of Mathematical Economics 16, 2 (April 1987), 191-200.

Ilya Segal. 2010. Personal communication. (2010).

Victor Shnayder, Jeremy Hoon, David Parkes, and Vikas Kawadia. 2012. Truthful Prioritization Schemes for Spectrum Sharing. In 7th Workshop on the Economics of Networks, Systems and Computation (NetEcon).

Aleksandrs Slivkins. 2011a. Contextual Bandits with Similarity Information. In 24th Conf. on Learning Theory (COLT). To appear in J. of Machine Learning Research (JMLR), 2014. 
Aleksandrs Slivkins. 2011b. Monotone multi-armed bandit allocations. Open Problem Session at COLT 2011 (Conf. on Learning Theory). (2011).

Aleksandrs Slivkins and Eli Upfal. 2008. Adapting to a Changing Environment: the Brownian Restless Bandits. In 21st Conf. on Learning Theory (COLT). 343-354.

Niranjan Srinivas, Andreas Krause, Sham Kakade, and Matthias Seeger. 2010. Gaussian Process Optimization in the Bandit Setting: No Regret and Experimental Design. In 27th Intl. Conf. on Machine Learning (ICML). 1015-1022.

John Thanassoulis. 2004. Haggling over substitutes. J. Economic Theory 117 (2004), 217-245.

Chris Wilkens and Balasubramanian Sivan. 2012. Single-Call Mechanisms. In 13th ACM Conf. on Electronic Commerce (EC). 